\title{
Numerical solution of a non-linear Volterra integral equation
}

Maleknejad, Khosrow ; Torabi, Parvin ; Sauter, Stefan A

\begin{abstract}
In this paper, a numerical method to solve non-linear integral equations based on a successive approximation technique is considered. A sequence of functions is produced which converges to the solution. The process includes a fixed point method, a quadrature rule, and an interpolation method. To find a total bound of the error, we investigate error bounds for each approximation and by combining them, we will derive an estimate for the total error. The accuracy and efficiency of the method is illustrated in some numerical examples.
\end{abstract}

DOI: https://doi.org/10.1007/s10013-015-0149-8

Posted at the Zurich Open Repository and Archive, University of Zurich ZORA URL: https://doi.org/10.5167/uzh-124961

Journal Article

Accepted Version

Originally published at:

Maleknejad, Khosrow; Torabi, Parvin; Sauter, Stefan A (2016). Numerical solution of a non-linear Volterra integral equation. Vietnam Journal of Mathematics, 44(1):5-28.

DOI: https://doi.org/10.1007/s10013-015-0149-8 


\title{
Numerical Solution of a Non-Linear Volterra Integral Equation
}

\author{
K. Maleknejad* \\ P. Torabi ${ }^{\dagger}$ \\ S. Sauter ${ }^{\ddagger}$ \\ Dedicated to Professor Eberhard Zeidler on the occasion of his 75th birthday
}

\begin{abstract}
In this paper, a numerical method to solve non-linear integral equations based on a successive approximation technique is considered. A sequence of functions is produced which converges to the solution. The process includes a fixed point method, a quadrature rule, and an interpolation method. To find a total bound of the error, we investigate error bounds for each approximation and by combining them, we will derive an estimate for the total error. The accuracy and efficiency of the method is illustrated in some numerical examples.
\end{abstract}

Keywords: Nonlinear quadratic Volterra integral equation; Fixed point theorem; Measure of noncompactness; Fixed point method; Adaptive quadrature; Nonuniform interpolation nodes.

\section{Introduction}

Integral equations play a very important role in nonlinear analysis and there are numerous applications in engineering, mathematical physics, economics, etc. (cf. $[18,1,9])$. Some problems considered in the vehicular traffic theory, biology, and queuing theory lead to the following nonlinear functional-integral equation:

$$
x(t)=f(t, x(t)) \int_{0}^{1} v(t, \tau, x(\tau)) d \tau,
$$

where $t \in I=[0,1]$ (cf.[11]). In this paper we will study the Volterra counterpart of the above equation denoted by

$$
x(t)=g(t)+f(t, x(t)) \int_{0}^{t} v(t, \tau, x(\tau)) d \tau,
$$

\footnotetext{
*School of Mathematics, Iran University of Science \& Technology, Narmak, Tehran 16846 13114, Iran, e-mail: maleknejad@iust.ac.ir

${ }^{\dagger}$ School of Mathematics, Iran University of Science \& Technology, Narmak, Tehran 16846 13114, Iran, e-mail: p_torabi@iust.ac.ir

${ }^{\ddagger}$ Institut für Mathematik, Universität Zürich, Winterthurerstrasse 190, CH-8057 Zürich, Switzerland, email: stas@math.uzh.ch
} 
where $t \in I=[0,1]$.

Existence of solutions for nonlinear integral equations, which contain particular cases of important integral and functional equations such as nonlinear Volterra integral equation, Urysohn integral equation and integral equations of Chandrasekhar type, have been considered in many papers and books [9],[3]-[15]. In [7] monotonicity properties of the superposition operator has been applied in the investigations of the solvability of a nonlinear quadratic integral equation of Volterra type. Also, authors have shown that under some assumptions the mentioned integral equation has monotonic and nonnegative (or positive) solutions in the space of real functions continuous on some bounded and closed interval.

Here, we investigate the smoothness property of the solution of the integral equation (1.1). Then we extend the successive approximation technique based on a fixed point method proposed in [16], and derive an error bound for the method. In Section 2 some preliminaries are introduced. Existence of the solution and fixed point method for the integral equation (1.1) are discussed in Sections 3 and 4. In Section 5, the smoothness of the solution is proved under appropriate assumptions on the smoothness of the data. Section 6 is devoted to the numerical solution based on a fixed point method, a quadrature rule, and an interpolation method. Then the convergence and error analysis of this numerical technique will be discussed in Section 7 . For this purpose, we derive bounds for quadrature and interpolation errors, separately. Then we will find a total error for the iterative method. Next, adaptive quadrature will be presented in Section 8 to reduce the cost of computations. Section 9 is devoted to numerical examples to illustrate the accuracy of the numerical method. Finally, in the Appendix we will prove some stability and convergence estimates for cubic spline interpolation under assumptions which allow for adaptive mesh refinement.

\section{Preliminaries}

Let us introduce some necessary tools which have been mentioned in ([7]). The superposition operator is one of the simplest nonlinear operator used in nonlinear functional analysis. On the other hand it is very important in the theory of integral and differential equations (cf. $[2])$. In order to define this operator assume that $J$ is a nonempty subset of the real line $\mathbb{R}$. Consider the set $X_{J}$ of real functions acting from the interval $[a, b]$ into the set $J$. Further, let $f:[a, b] \times J \rightarrow \mathbb{R}$ be a given function. Then, to every function $x \in X$ we may assign the function $F x$ defined by the formula

$$
(F x)(t):=f(t, x(t)), \quad t \in[a, b] .
$$

The operator $F$ defined in such a way is called the superposition operator generated by the function $f=f(t, x)$.

The main tools used in our considerations are the concept of a measure of noncompactness and the concept of degree of monotonicity of a real function. In order to present the first concept mentioned above suppose that $E$ is a real Banach space with norm $\|\cdot\|$. For a given nonempty subset $X$ of $E$ denote by $\bar{X}$, conv $(X)$ the closure and the closed convex hull of $X$, respectively. Further, let $M_{E}$ denote the family of all nonempty and bounded subsets of $E$ and by $N_{E}$ its subfamily consisting of all relatively compact sets. We recall the following definition from [3].

Definition 2.1 A mapping $\mu: M_{E} \rightarrow \mathbb{R}^{+}=[0,+\infty)$ is said to be the measure of noncompactness in $E$, if it satisfies the following conditions 
(1) the family $\operatorname{ker}(\mu)=\left\{X \in M_{E}: \mu(X)=0\right\}$ is nonempty and $\operatorname{ker}(\mu) \subset N_{E}$;

(2) $X \subseteq Y \Rightarrow \mu(X) \leq \mu(Y)$;

(3) $\mu(\bar{X})=\mu(\operatorname{conv}(X))=\mu(X)$;

(4) $\mu(\lambda X+(1-\lambda) Y) \leq \lambda \mu(X)+(1-\lambda) \mu(Y)$ for $\lambda \in[0,1]$;

(5) if $\left(X_{n}\right)$ is a sequence of closed sets from $M_{E}$ such that $X_{n+1} \subset X_{n}$ for $n=1,2, \ldots$, and if $\lim _{n \rightarrow \infty} \mu\left(X_{n}\right)=0$, then the set $X_{\infty}=\bigcap_{n=1}^{\infty} X_{n}$ is nonempty.

A measure $\mu$ is said to be sublinear if it satisfies the following two conditions:

(6) $\mu(\lambda X)=|\lambda| \mu(X)$ for $\lambda \in \mathbb{R}$;

(7) $\mu(X+Y) \leq \mu(X)+\mu(Y)$.

The family $\operatorname{ker}(\mu)$ described in Definition 2.1(1) is called the kernel of the measure of noncompactness $\mu$. More information about measures of noncompactness and their properties can be found in [3]. For our purposes we will only need the following fixed point theorem [3].

Theorem 2.2 Let $Q$ be a nonempty bounded closed convex subset of the Banach space $E$ and let $T: Q \rightarrow Q$ be a continuous mapping and $\mu(X)$ be the measure of noncompactness in $E$. Assume that there exists a constant $k \in[0,1)$ such that $\mu(T X) \leq k \mu(X)$ for any nonempty subset $X$ of $Q$. Then $T$ has a fixed point in the set $Q$.

In what follows let $I=[0,1]$ be a fixed interval in $\mathbb{R}$. Denote by $C=C(I)$ the classical Banach space of all continuous real functions on $I$ equipped with the standard norm $\|x\|=$ $\max \{|x(t)|: t \in I\}$. Now, let us fix a set $X \in M_{C}$. For $x \in X$ let us define the following quantities (cf. [5]):

$$
d(x):=\sup \{|x(s)-x(t)|-[x(s)-x(t)]: t, s \in I, t \leq s\} .
$$

Analogously, put

$$
d(X):=\sup \{d(x): x \in X\} .
$$

Observe that $d(x)=0$ if and only if $x$ is nondecreasing on $I$. Similarly, $d(X)=0$ if and only if all functions belonging to $X$ are nondecreasing on $I$. Thus the index $d(x)$ represents the degree of decrease of the function $x$ on $I$. Analogously, the quantity $d(X)$ measures the degree of decrease of functions from the set $X$.

In what follows $\varepsilon>0$ and denote by $\omega(x, \varepsilon)$ the modulus of continuity of the function $x$, i.e.

$$
\omega(x, \varepsilon):=\sup \{|x(s)-x(t)|: t, s \in I,|t-s| \leq \varepsilon\} .
$$

Similarly, let us put

$$
\omega(X, \varepsilon):=\sup \{\omega(x, \varepsilon): x \in X\} \quad \text { and } \quad \omega_{0}(X):=\lim _{\varepsilon \rightarrow 0} \omega(X, \varepsilon) .
$$

Finally, the function $\mu$ is defined on the family $M_{C}$ by putting

$$
\mu(X)=\omega_{0}(X)+d(X) .
$$

It can be shown [5] that the function $\mu$ is a measure of noncompactness in the space $C(I)$ with the kernel ker $(\mu)$ consisting of all nonempty and bounded sets $X$ such that functions from $X$ are equicontinuous and nondecreasing on $I$. For other properties of $\mu$ see [5]. 


\section{Existence of the Solution}

For the nonlinear Volterra integral equation (1.1), existence of the monotonic solution has been proved by Banaś and Sadarangani [7] and we sketch, for convenience, the main ideas of the general theory.

Assume that $f$ is a real function defined on the set $I \times J$, where $J$ is an arbitrary real interval. We consider the superposition operator $(F x)(t)=f(t, x(t))$ under the following assumptions.

\section{Assumption 3.1}

( $\alpha$ ) The function $f$ is continuous on the set $I \times J$.

$(\beta)$ The function $t \rightarrow f(t, x)$ is nondecreasing on $I$ for any fixed $x \in J$.

$(\gamma)$ For any fixed $t \in I$ the function $x \rightarrow f(t, x)$ is nondecreasing on $J$.

( $\delta)$ The function $f=f(t, x)$ satisfies the Lipschitz condition with respect to the variable $x$, i.e. there exists a constant $k>0$ such that for any $t \in I$ and for $x_{1}, x_{2} \in J$ the following inequality holds

$$
\left|f\left(t, x_{2}\right)-f\left(t, x_{1}\right)\right| \leq k\left|x_{2}-x_{1}\right|
$$

Then the following result is implied.

Theorem 3.2 Let Assumption 3.1 be satisfied and $x \in X_{J} \subseteq C(I)$. Then

$$
d(F x) \leq k d(x) .
$$

For a proof, we refer to [7].

The above theorem implies for any function $f$ which satisfies the Lipschitz condition with a constant $k<1$ (cf. the Assumption 3.1( $\delta)$ ) that the superposition operator $F$, generated by the function $f$, improves the degree of monotonicity of any subset $X$ of $X_{J}$ with the coefficient $k$.

Corollary 3.3 Suppose the function $f(t, x)=f: I \times J \rightarrow \mathbb{R}$ satisfies Assumption 3.1 $(\alpha),(\beta)$. Moreover, we assume that the partial derivative $\partial_{2} f$ of $f$ exists, is nonnegative, and bounded on the set $I \times J$. Then $f$ satisfies the assumptions $(\gamma)$ and $(\delta)$ with the Lipschitz constant $k$ defined by

$$
k:=\sup \left\{\partial_{2} f(t, x):(t, x) \in I \times J\right\} .
$$

Using the measure of noncompactness and the results established in this section, Banaś and Sadarangani in [7] showed that (1.1) has monotonic and nonnegative solutions under the following assumption.

\section{Assumption 3.4}

(i) $g \in C(I)$ and $g$ is nondecreasing and nonnegative on the interval $I$.

(ii) The function $f: I \times J \rightarrow \mathbb{R}$ satisfies the conditions $(\alpha)-(\delta)$, where $J \subset \mathbb{R}^{+}$is an unbounded interval and $g_{0} \in J$, where $g_{0}=g(0)=\min \{g(t): t \in I\}$. Moreover, $f$ is nonnegative on $I \times J$. 
(iii) There exists a nondecreasing function $k(r)=k:\left[g_{0},+\infty\right) \rightarrow \mathbb{R}^{+}$such that

$$
\left|f\left(t, x_{1}\right)-f\left(t, x_{2}\right)\right| \leq k(r)\left|x_{1}-x_{2}\right|
$$

for any $t \in I$ and for all $x_{1}, x_{2} \in\left[g_{0}, r\right]$.

(iv) $v: I \times I \times \mathbb{R} \rightarrow \mathbb{R}$ is a continuous function such that $v: I \times I \times \mathbb{R}^{+} \rightarrow \mathbb{R}^{+}$and for arbitrarily fixed $\tau \in I$ and $x \in \mathbb{R}^{+}$the function $t \rightarrow v(t, \tau, x)$ is nondecreasing on $I$.

(v) There exists a nondecreasing function $p: \mathbb{R}^{+} \rightarrow \mathbb{R}^{+}$such that

$$
v(t, \tau, x) \leq p(x)
$$

for $t, \tau \in I$ and $x \geq 0$.

(vi) There exists a positive solution $r_{0}$ for the inequality

$$
\|g\|+\left(r k(r)+F_{0}\right) p(r) \leq r,
$$

where $F_{0}=\|f(\cdot, 0)\|$. Moreover, $k\left(r_{0}\right) p\left(r_{0}\right)<1$.

This assumption implies the existence of a solution as stated in the next theorem.

Theorem 3.5 Let Assumption 3.4 be satisfied. Then, equation (1.1) has at least one solution $x=x(t)$ which belongs to the space $C(I)$ and is nondecreasing and nonnegative on the interval $I$.

For a proof, we refer to [7].

\section{Fixed Point Method and its Convergence}

In this section, we present some assumptions to guarantee the unique solvability of equation (1.1) and then show that the fixed point method converges to this solution. For this purpose we follow the notation in [7]. Let us take the subset $S$ of the space $C(I), S=\{x \in C(I)$ : $x(t) \geq g_{0}=g(0)$ for $\left.t \in I\right\}$, and define the operator $T$ on the set $S$ by the formula ${ }^{1}$

$$
T x:=g(t)+T_{0}(f, v, x) \quad \text { with } \quad\left(T_{0}(h, w, x)\right)(t):=h(t, x(t)) \int_{0}^{t} w(t, \tau, x(\tau)) d \tau .
$$

From the proof of Theorem 3.5 it follows that $T$ transforms the set $S$ into itself as well as the set

$$
S_{r_{0}}=\left\{x \in S:\|x\| \leq r_{0}\right\},
$$

where $r_{0}>0$ is as in Assumption 3.4(vi). $S_{r_{0}}$ is nonempty. Since $r_{0} \geq g_{0}$, and $S_{r_{0}}$ is a bounded, closed, and convex subset of $C(I)$, also $T$ is continuous on the set $S_{r_{0}}$ and has at least one fixed point in this set.

We choose the initial function $x_{0} \in S_{r_{0}}$ and generate the sequence $\left(x_{n}(t)\right)_{n=0}^{\infty}$ by the recursion

$$
x_{n+1}(t):=\left(T x_{n}\right)(t)=g(t)+f\left(t, x_{n}(t)\right) \int_{0}^{t} v\left(t, \tau, x_{n}(\tau)\right) d \tau, \quad t \in I, n \geq 0 .
$$

We show in the following theorem that under the given assumptions the sequence $\left(x_{n}(t)\right)_{n=0}^{\infty}$ generated by $T: S_{r_{0}} \rightarrow S_{r_{0}}$ converges to the unique fixed point $\widetilde{x}(t)$ of $T$.

\footnotetext{
${ }^{1}$ For $h=f$ and $w=v$ we write $T_{0} x$ short for $T_{0}(f, v, x)$.
} 
Theorem 4.1 Let the function $T: E \rightarrow E$ have a fixed point $x$, i.e., $T(x)=x$. Further let $S_{r}(x)=\{y \in E:\|y-x\|<r\}$ be a neighborhood of $x$ such that $T$ is a contractive mapping in $S_{r}(x)$, that is,

$$
\|T(x)-T(y)\| \leq L\|x-y\|, \quad 0<L<1,
$$

for all $x, y \in S_{r}(x)$. Then for any $x_{0} \in S_{r}(x)$ the sequence $x_{n+1}=T\left(x_{n}\right), n=0,1, \ldots$, has the properties

(a) $x_{n} \in S_{r}(x)$ for all $n=0,1, \ldots$

(b) $\left\|x_{n+1}-x\right\| \leq L\left\|x_{n}-x\right\| \leq L^{n+1}\left\|x_{0}-x\right\|$,

as well as

$$
\left\|x_{n}-x\right\| \leq \frac{L^{n}}{1-L}\left\|x_{1}-x_{0}\right\|
$$

i.e., $\left(x_{n}\right)_{n}$ converges at least linearly to $x$.

For a proof, we refer, e.g., to [19].

Theorem 4.2 Let the operator $T$, as in (4.1), satisfy the following assumptions

(a) The function $v(t, \tau, x)$ satisfies the Lipschitz condition with respect to variable $x$ with constant $\vartheta>0$, i.e.,

$$
\forall t, \tau \in I \quad \forall x_{1}, x_{2} \in S_{r_{0}} \quad\left|v\left(t, \tau, x_{2}\right)-v\left(t, \tau, x_{1}\right)\right| \leq \vartheta\left|x_{2}-x_{1}\right| .
$$

(b) $r_{0}$ satisfies Assumption 3.4(vi) as well as the following inequality

$$
p\left(r_{0}\right) k\left(r_{0}\right)+\left(r_{0} k\left(r_{0}\right)+F_{0}\right) \vartheta<1 .
$$

This relation implies $k\left(r_{0}\right) p\left(r_{0}\right)<1$ (mentioned in Assumption 3.4(vi)) automatically.

Then the operator $T$ is a contractive mapping in $S_{r_{0}}$, so it has exactly one fixed point and the generated sequence $\left(x_{n}(t)\right)_{n=0}^{\infty}$ is converging to this fixed point, i.e.

$$
\lim _{n \rightarrow \infty} x_{n}(t)=x(t), \quad \forall t \in I,
$$

where $(T x)(t)=x(t)$, and

$$
\left\|x_{n}-x\right\| \leq \frac{L^{n}}{1-L}\left\|x_{1}-x_{0}\right\|
$$

where $0<L<1$.

Proof. Suppose that $x, y \in S_{r_{0}}$, then for $t \in I$ it holds

$$
\begin{aligned}
|(T x)(t)-(T y)(t)| \leq & \left|f(t, x(t)) \int_{0}^{t} v(t, s, x(s)) d s-f(t, y(t)) \int_{0}^{t} v(t, s, y(s)) d s\right| \\
\leq & \left|f(t, x(t)) \int_{0}^{t} v(t, s, x(s)) d s-f(t, y(t)) \int_{0}^{t} v(t, s, x(s)) d s\right| \\
& +\left|f(t, y(t)) \int_{0}^{t} v(t, s, x(s)) d s-f(t, y(t)) \int_{0}^{t} v(t, s, y(s)) d s\right| \\
\leq & k\left(r_{0}\right)\|x(t)-y(t)\| \int_{0}^{t} v(t, s, x(s))+f(t, y(t)) \int_{0}^{t}|v(t, s, x(s))-v(t, s, y(s))| d s \\
\leq & p\left(r_{0}\right) k\left(r_{0}\right)\|x(t)-y(t)\|+\left(r_{0} k\left(r_{0}\right)+F_{0}\right) \vartheta\|x(t)-y(t)\| \\
= & \left(p\left(r_{0}\right) k\left(r_{0}\right)+\left(r_{0} k\left(r_{0}\right)+F_{0}\right) \vartheta\right)\|x(t)-y(t)\| .
\end{aligned}
$$


Let $L:=p\left(r_{0}\right) k\left(r_{0}\right)+\left(r_{0} k\left(r_{0}\right)+F_{0}\right) \vartheta$. From Assumption (b) we conclude that $L<1$ holds. Hence the operator $T$ satisfies the Lipschitz condition, i.e.

$$
\|T x-T y\| \leq L\|x-y\| .
$$

Now, suppose $\widetilde{x}, \widetilde{y} \in S_{r_{0}}$ be the fixed points of $T$. Then

$$
\|\widetilde{x}-\widetilde{y}\|=\|T \widetilde{x}-T \widetilde{y}\| \leq L\|\widetilde{x}-\widetilde{y}\| .
$$

From $0<L<1$, one concludes that $\widetilde{x} \equiv \widetilde{y}$ holds. Hence, $T$ has a unique fixed point in $S_{r_{0}}$. The combination of (4.8) with Theorem 4.1 implies (4.6) and (4.7) with $E=C(I)$.

Remark 4.3 Assume that $v(t, s, x)$ has a continuous partial derivative $\frac{\partial v}{\partial x}$ in its domain $D_{v}=$ $[0,1] \times[0,1] \times J$. Then $v$ is Lipschitz continuous in $x$.

\section{Properties of the Solution}

In this section, we prove that for $m$-times differentiable functions $g(t), f(t, x)$, and $v(t, s, x)$, the corresponding solution $x(t)$ has $m$ derivatives, too. For $d \in \mathbb{N}$ and $\ell \in \mathbb{N}_{0}$, we introduce the index set

$$
\iota_{\ell}^{d}:=\left\{\mu \in \mathbb{N}_{0}^{d}|| \mu \mid \leq \ell\right\} \quad \text { with }|\mu|=\sum_{i=1}^{d} \mu_{i},
$$

while, for $d_{1}, d_{2} \in \mathbb{N}$, we put

$$
\iota_{\ell}^{d_{1}, d_{2}}:=\left\{(\mu, \nu) \in \iota_{\ell}^{d_{1}} \times \iota_{\ell}^{d_{2}}|| \mu|+| \nu \mid \leq \ell\right\} .
$$

For a $d$-dimensional domain $D \subset \mathbb{R}^{d}$ and a function $q \in C^{\ell}(D)$, we set

$$
|q|_{\ell}:=\max _{\substack{\mu \in i_{\ell}^{d} \\|\mu|=\ell}}\left\|\partial^{\mu} q\right\| \quad \text { and } \quad\|q\|_{\ell}:=\max _{\mu \in \iota_{\ell}^{d}}\left\|\partial^{\mu} q\right\| .
$$

Theorem 5.1 Let Assumption 3.4 be satisfied and let $g \in C^{m}(I), f \in C^{m}(I \times J), v \in$ $C^{m}\left(I^{2} \times J\right)$. Assume

$$
\left\|\partial_{2} f\right\|\|v\|<1
$$

Then $x \in C^{m}(I)$, and

$$
|x|_{m} \leq \frac{|g|_{m}+\|f\|_{m}\|v\|_{m} \check{p}_{m}\left(\|x\|_{m-1}\right)}{1-\left\|\partial_{2} f\right\|\|v\|}
$$

with a polynomial $\check{p}_{m}$. Thus,

$$
|x|_{m} \leq \psi_{m}\left(\|g\|_{m},\|f\|_{m},\|v\|_{m}, \frac{1}{1-|f|_{1}|v|_{0}}\right),
$$

where $\psi_{m}$ is a polynomial in each variable. 
Proof. According to Theorem 3.5, we know $x \in C(I)$. Then by induction, suppose that (5.2) holds for all $j=0, \ldots, m-1$. We write the equation in the following way:

$$
x=g+F \mathcal{V}
$$

where

$$
\mathcal{V}(t)=\int_{0}^{t} v(t, s, x(s)) d s \quad \text { and } \quad F:=f(\cdot, x(\cdot)) .
$$

By using Leibniz' rule for the $m$-th derivative of a product we obtain from (5.4)

$$
x^{(m)}=g^{(m)}+\sum_{\ell=0}^{m}\left(\begin{array}{c}
m \\
\ell
\end{array}\right) F^{(m-\ell)} \mathcal{V}^{(\ell)} .
$$

It is easy to see via induction that for $0 \leq \ell \leq m$

$$
F^{(\ell)}=\left(\partial_{1}^{\ell} f\right)(\cdot, x(\cdot))+\sum_{s=1}^{\ell} \sum_{i=0}^{s-1} p_{\ell, s, i}\left(x^{\prime}, \ldots, x^{(\ell+1-s)}\right)\left(\partial_{1}^{i} \partial_{2}^{s-i} f\right)(\cdot, x(\cdot)),
$$

where $p_{\ell, s, i}$ is a polynomial in each variable. In particular, we have $p_{\ell, 1,0}\left(x^{\prime}, \ldots, x^{(\ell)}\right)=x^{(\ell)}$ so that for $\ell \geq 1$

$$
F^{(\ell)}=x^{(\ell)} \partial_{2} f(\cdot, x(\cdot))+\mathcal{R}_{\ell}\left(\left(x^{(r)}\right)_{r=1}^{\ell-1},\left(\left(\partial^{\mu} f\right)(\cdot, x(\cdot))\right)_{\mu \in \iota_{\ell}^{2}}\right),
$$

where, for $\mathbf{x}=\left(x_{r}\right)_{r=1}^{\ell-1}$

$\mathcal{R}_{\ell}\left(\mathbf{x},\left(\left(\partial^{\mu} f\right)(\cdot, x(\cdot))\right)_{\mu \in \iota_{\ell}^{2}}\right):=\left(\partial_{1}^{\ell} f\right)(\cdot, x(\cdot))+\sum_{s=2}^{\ell} \sum_{i=0}^{s-1} p_{\ell, s, i}\left(x^{\prime}, \ldots, x^{(\ell+1-s)}\right)\left(\partial_{1}^{i} \partial_{2}^{s-i} f\right)(\cdot, x(\cdot))$

is a polynomial in each variable which contains only derivatives of $x$ up to an order $\ell-1$. Note that $\mathcal{R}_{\ell}$ is linear with respect to $f$, i.e., for $\mathbf{x}=\left(x_{r}\right)_{r=1}^{\ell-1}$

$$
\begin{aligned}
\mathcal{R}_{\ell}\left(\mathbf{x},\left(\left(\partial^{\mu}\left(\lambda f_{1}+f_{2}\right)\right)(\cdot, x(\cdot))\right)_{\mu \in \iota_{\ell}^{2}}\right) & =\lambda \mathcal{R}_{\ell}\left(\mathbf{x},\left(\left(\partial^{\mu} f_{1}\right)(\cdot, x(\cdot))\right)_{\mu \in \iota_{\ell}^{2}}\right) \\
& +\mathcal{R}_{\ell}\left(\mathbf{x},\left(\left(\partial^{\mu}\left(f_{2}\right)\right)(\cdot, x(\cdot))\right)_{\mu \in \iota_{\ell}^{2}}\right) .
\end{aligned}
$$

Next, we will consider the $\ell$-th derivatives of $\mathcal{V}$. We set $w_{k}(t)=\partial_{1}^{k} v(t, t, x(t))$ and write

$$
\mathcal{V}^{(\ell)}(t)=\sum_{k=0}^{\ell-1} w_{k}^{(\ell-1-k)}(t)+\int_{0}^{t} \partial_{1}^{\ell} v(t, s, x(s)) d s .
$$

We set $W_{k}(t, y):=\partial_{1}^{k} v(t, t, y)$. As in (5.7a) we get

$$
w_{k}^{(j)}=\left(\partial_{1}^{j} W_{k}\right)(\cdot, x(\cdot))+\sum_{s=1}^{j} \sum_{i=0}^{s-1} p_{j, s, i}\left(x^{\prime}, \ldots, x^{(j+1-s)}\right)\left(\partial_{1}^{i} \partial_{2}^{s-i} W_{k}\right)(\cdot, x(\cdot)) .
$$

On the other hand, we have

$$
\partial_{1}^{i} \partial_{2}^{j} W_{k}(t, y)=\nabla_{12}^{i} \partial_{3}^{j} w_{k}(t, t, y) \quad \text { with } \quad \nabla_{12}^{i}=\sum_{r=0}^{i}\left(\begin{array}{l}
i \\
r
\end{array}\right) \partial_{1}^{r} \partial_{2}^{i-r}
$$


so that

$$
w_{k}^{(j)}=\partial_{1}^{k} \nabla_{12}^{j} v(\cdot, \cdot, x(\cdot))+\sum_{s=1}^{j} \sum_{i=0}^{s-1} p_{j, s, i}\left(x^{\prime}, \ldots, x^{(j+1-s)}\right)\left(\partial_{1}^{k} \nabla_{12}^{i} \partial_{3}^{s-i} v\right)(\cdot, \cdot, x(\cdot)) .
$$

This leads to the representation

$$
\mathcal{V}^{(\ell)}=\kappa_{\ell}\left(\left(x^{(r)}\right)_{r=1}^{\ell-1},\left(\partial^{\mu} v(\cdot, \cdot, x(\cdot))\right)_{\mu \in \iota_{\ell-1}^{3}}\right)+\int_{0}^{\cdot} \partial_{1}^{\ell} v(\cdot, s, x(s)) d s,
$$

where $\kappa_{\ell}$ is a polynomial in each variable $x^{(r)}$ and linear with respect to $v$ (in an analogous way as $(5.7 \mathrm{~d}))$.

The representations for $\mathcal{V}^{(\ell)}$ and $F^{(\ell)}$ allow for a representation of $x^{(m)}$. We introduce the operator

$$
H_{0}(f, v, x):=f(\cdot, x(\cdot)) v(\cdot, \cdot, x(\cdot)) .
$$

Then, it holds for $\ell \geq 1$

$$
\begin{aligned}
(g+F \mathcal{V})^{(\ell)} & =g^{(\ell)}+x^{(\ell)} T_{0}\left(\partial_{2} f, v, x\right) \\
& +q_{\ell}^{\mathrm{I}}\left(\left(H_{0}\left(\partial^{\mu} f, \partial^{\nu} v, x\right)\right)_{(\mu, \nu) \in \iota_{\ell-1}^{2,3}},\left(x^{(r)}\right)_{r=1}^{\ell-1}\right) \\
& +q_{\ell}^{\mathrm{II}}\left(T_{0}\left(\partial^{\mu} f, \partial_{1}^{\nu} v\right)_{(\mu, \nu) \in \iota_{\ell}^{2,1}},\left(x^{(r)}\right)_{r=1}^{\ell-1}\right),
\end{aligned}
$$

where $q_{\ell}^{\mathrm{I}, \mathrm{II}}$ are polynomials with respect to each argument $x^{(r)}$ while they are linear with respect to the first arguments:

$$
\begin{aligned}
q_{\ell}^{\mathrm{I}}\left(\left(H_{0}\left(\partial^{\mu}\left(\lambda f_{1}+f_{2}\right), \partial^{\nu} v\right)\right)_{(\mu, \nu) \in \iota_{\ell-1}^{2,3}}, \mathbf{x}\right) & =\lambda q_{\ell}^{\mathrm{I}}\left(\left(H_{0}\left(\partial^{\mu} f_{1}, \partial^{\nu} v\right)\right)_{(\mu, \nu) \in \iota_{\ell-1}^{2,3}}, \mathbf{x}\right) \\
& +q_{\ell}^{\mathrm{I}}\left(\left(H_{0}\left(\partial^{\mu}\left(f_{2}\right), \partial^{\nu} v\right)\right)_{(\mu, \nu) \in \iota_{\ell-1}^{2,3}}, \mathbf{x}\right)
\end{aligned}
$$

Analogous relations hold if $v$ is replaced by $\lambda v_{1}+v_{2}$ and also for $q_{\ell}^{\mathrm{II}}$. Hence, there exist polynomials $p_{\ell}^{\mathrm{I}}(\mathbf{z})$ and $p_{\ell}^{\mathrm{II}}(\mathbf{z})$ with $\mathbf{z}=\left(z_{r}\right)_{r=1}^{\ell-1}$ such that for $\boldsymbol{\xi}=\left(\xi_{\mu, \nu}\right)_{(\mu, \nu) \in \iota_{\ell-1}^{2,3}}$ and $\boldsymbol{\zeta}=$ $\left(\zeta_{\mu, \nu}\right)_{(\mu, \nu) \in \iota_{\ell}^{2,1}}$ it holds

$$
\begin{aligned}
q_{\ell}^{\mathrm{I}}(\boldsymbol{\xi}, \mathbf{z}) & \leq\|\boldsymbol{\xi}\| p_{\ell}^{\mathrm{I}}(\mathbf{z}) \\
\left|q_{\ell}^{\mathrm{II}}\left(\boldsymbol{\zeta},\left(z_{r}\right)_{r=1}^{\ell-1}\right)\right| & \leq\|\boldsymbol{\zeta}\| p_{\ell}^{\mathrm{II}}(\mathbf{z}) .
\end{aligned}
$$

Hence, the estimate

$$
|g+F \mathcal{V}|_{\ell} \leq|g|_{\ell}+|x|_{\ell}\left\|\partial_{2} f\right\|\|v\|+\|f\|_{\ell}\|v\|_{\ell} \tilde{p}_{\ell}\left(\|x\|_{\ell-1}\right)
$$

follows for some polynomial $\tilde{p}_{\ell}$. This implies

$$
|x|_{\ell} \leq \frac{1}{1-\left\|\partial_{2} f\right\|\|v\|}\left(|g|_{\ell}+\|f\|_{\ell}\|v\|_{\ell} \check{p}_{\ell}\left(\|x\|_{\ell-1}\right)\right) .
$$

and the assertion follows by induction. 


\section{Numerical Solution Method}

In this section, we will present a fully discrete fixed point method for the integral equation (1.1). We approximate the function $x(t)$ by cubic spline collocation and employ a quadrature method to approximate the integral.

Continuous non-linear Volterra integral equation of second kind:

$$
x(t)=g(t)+f(t, x(t)) \int_{0}^{t} v(t, s, x(s)) d s, \quad t \in I=[0,1] .
$$

Fixed point iteration: By choosing the initial function $x_{0}=g$, the corresponding fixed point iteration is:

$$
x_{n+1}(t)=g(t)+f\left(t, x_{n}(t)\right) \int_{0}^{t} v\left(t, s, x_{n}(s)\right) d s,
$$

for $n=0,1, \ldots$, which converges to the solution under the assumptions as stated in Theorem 4.2 .

Spline collocation: We choose collocation points $t_{0}=0<t_{1}<\cdots<t_{n-1}<t_{N}=1$ and define a partition $\Delta=\left\{\tau_{1}, \tau_{2}, \ldots, \tau_{N}\right\}$ of the interval $I$ into subintervals $\tau_{i}=\left(x_{i-1}, x_{i}\right)$, $1 \leq i \leq N$. The space of cubic spline functions subordinate to $\Delta$ is denoted by $S_{\Delta}$. For nodal values $\mathbf{w}^{(n)}=\left(w_{i}^{(n)}\right)_{i=0}^{N}$ at time points $t_{n}$ we denote the cubic spline interpolation operator by $I_{\Delta}$. Then, the fixed point iteration with spline collocation is given by setting $x_{0}^{\Delta}=g$ and determining, for $n=0,1, \ldots$, the functions $x_{n+1}^{\Delta}=I_{\Delta}\left(\mathbf{x}^{(n+1)}\right) \in S_{\Delta}$ by their nodal values

$$
x_{i}^{(n+1)}:=g\left(t_{i}\right)+f\left(t_{i}, x_{i}^{(n)}\right) \int_{0}^{t_{i}} v\left(t_{i}, s, x_{n}^{\Delta}(s)\right) d s, \quad i=0, \ldots, N .
$$

Quadrature Approximation: To approximate the integral in (6.2) we use the following quadrature rule. Let $Q(u)=\sum_{j=1}^{n_{Q}} w_{j} u\left(\xi_{j}\right)$ be a fixed quadrature rule to approximate the integral $\int_{-1}^{1} u$ and let $Q_{[a, b]}$ denote the scaled version for the interval $[a, b]$, i.e., $Q_{[a, b]}(u):=$ $\frac{b-a}{2} \sum_{j=1}^{n_{Q}} w_{j} u\left(\frac{1-\xi_{j}}{2} a+\frac{1+\xi_{j}}{2} b\right)$. For $\left.\left.t \in\right] 0,1\right]$, define $i=i(t)$ by $\left.\left.t \in\right] t_{i-1}, t_{i}\right]$ and define the quadrature approximation by

$$
\int_{0}^{t} v(t, s, x(s)) d s \approx Q_{t}(x):=\sum_{j=1}^{i} Q_{\widetilde{\tau}_{j}}(v(t, \cdot, x(\cdot))), \quad \text { where } \tilde{\tau}_{j}:= \begin{cases}\tau_{j} & j<i, \\ {\left[t_{i-1}, t\right]} & j=i .\end{cases}
$$

We use the short hands $Q_{i}, w_{i, j}, s_{i, j}, n_{i}$ if $t=t_{i}$ is a collocation point. By substituting this approximation into equation (6.2) we obtain the following recursion;

$$
\begin{array}{ll}
\text { Initialization: } & x_{0}^{\mathrm{Q}, \Delta}=g \\
\text { for } n=0,1, \ldots & X_{i}^{(n+1)}:=g\left(t_{i}\right)+f\left(t_{i}, X_{i}^{(n)}\right) Q_{i}\left(x_{n}^{\mathrm{Q}, \Delta}\right), \quad i=0, \ldots, N \\
& x_{n+1}^{Q, \Delta}:=I_{\Delta}\left(\mathbf{X}^{(n+1)}\right) .
\end{array}
$$

\section{Error Analysis}

In this section, we will derive a bound for the total error by analyzing the three approximation steps (fixed point iteration/spline collocation/quadrature). First we are concerned with the 
convergence of the method and start to prove a version of Theorem 4.2 for the fully discrete case. We introduce

$$
\mathbf{g}=\left(g\left(t_{i}\right)\right)_{i=0}^{N} \quad \text { and } \quad \mathbf{Q}(x):=\mathbf{g}+\mathbf{Q}_{0}(x) \quad \text { with } \quad \mathbf{Q}_{0}(x):=\left(f\left(t_{i}, x\left(t_{i}\right)\right) Q_{i}(x)\right)_{i=0}^{N} .
$$

so that the iteration (6.4) can be written in the form: $x_{0}^{\mathrm{Q}, \Delta}:=g$ and

$$
x_{n+1}^{\mathrm{Q}, \Delta}:=I_{\Delta} \mathbf{Q}\left(x_{n}^{\mathrm{Q}, \Delta}\right) .
$$

Theorem 7.1 Let (4.5) and Assumption 3.4 be valid. Let

$$
C_{\Delta}:=\sup _{\mathbf{v} \in \mathbb{R}^{N+1} \backslash\{0\}} \frac{\left\|I_{\Delta} \mathbf{v}\right\|}{\|\mathbf{v}\|_{\max }} \quad \text { and } \quad C_{\mathrm{Q}}:=\max _{1 \leq i \leq N} \frac{\sum_{j=0}^{n_{i}}\left|w_{i, j}\right|}{\sum_{j=0}^{n_{i}} w_{i, j}}
$$

and assume (cf. Assumptions a,b of Theorem 4.2)

$$
L_{\Delta}:=C_{\Delta} C_{\mathrm{Q}}\left(k\left(r_{0}\right) p\left(r_{0}\right)+\left(r_{0} k\left(r_{0}\right)+F_{0}\right) \vartheta\right)<1 .
$$

Then, the operator $I_{\Delta} \mathbf{Q}$ is a contraction in $S_{r_{0}}$, so it has exactly one fixed point and the generated sequence $\left(x_{n}^{\mathrm{Q}, \Delta}\right)_{n}$ (cf. (7.2)) is converging to this fixed point, i.e.,

$$
\lim _{n \rightarrow \infty} x_{n}^{\mathrm{Q}, \Delta}=x^{\mathrm{Q}, \Delta},
$$

where $x^{\mathrm{Q}, \Delta}=I_{\Delta} \mathbf{Q}\left(x^{\mathrm{Q}, \Delta}\right)$ and

$$
\left\|x_{n}^{\mathrm{Q}, \Delta}-x^{\mathrm{Q}, \Delta}\right\| \leq \frac{L^{n}}{1-L}\left\|x_{1}^{\mathrm{Q}, \Delta}-x_{0}^{\mathrm{Q}, \Delta}\right\| \quad \text { for some } 0<L<1 .
$$

Remark 7.2 In Theorem A.1, we will prove that $C_{\Delta}$ can be bounded always in terms of the global quasi-uniformity of the mesh or, alternatively, under some mild assumptions, in terms of the local quasi-uniformity of the mesh.

Proof of Theorem 7.1. Let $x, y \in S_{r_{0}}$. It holds

$$
\left\|\left(I_{\Delta} \mathbf{Q}\right)(x)-\left(I_{\Delta} \mathbf{Q}\right)(y)\right\| \leq C_{\Delta}\|\mathbf{Q}(x)-\mathbf{Q}(y)\|_{\max } .
$$

The difference in the right-hand side can be written in the form

$$
\begin{aligned}
(\mathbf{Q}(x)-\mathbf{Q}(y))_{i} & =\left(f\left(t_{i}, x\left(t_{i}\right)\right)-f\left(t_{i}, y\left(t_{i}\right)\right)\right) Q_{i}(x) \\
& +\left(f\left(t_{i}, y\left(t_{i}\right)\right)-f\left(t_{i}, 0\right)+f\left(t_{i}, 0\right)\right)\left(Q_{i}(x)-Q_{i}(y)\right) .
\end{aligned}
$$

From (3.1) and (3.3), we conclude that

$$
\begin{aligned}
\left|(\mathbf{Q}(x)-\mathbf{Q}(y))_{i}\right| & \leq k\left(r_{0}\right)\|x-y\|\left|Q_{i}(x)\right| \\
& +\left(r_{0} k\left(r_{0}\right)+F_{0}\right)\left|Q_{i}(x)-Q_{i}(y)\right| .
\end{aligned}
$$

Next we estimate the terms related to the quadrature method. It holds

$$
\left|Q_{i}(x)\right|=\left|\sum_{j=0}^{n_{i}} w_{i, j} v\left(t, s_{i, j}, x\left(s_{i, j}\right)\right)\right| \stackrel{(3.2)}{\leq} C_{\mathrm{Q}} p\left(r_{0}\right)
$$


with the stability constant $C_{\mathrm{Q}}$ of the quadrature method. Furthermore, we get

$$
\left|Q_{i}(x)-Q_{i}(y)\right| \leq \sum_{j=0}^{n_{i}}\left|w_{i, j}\right|\left|v\left(t, s_{i, j}, x\left(s_{i, j}\right)\right)-v\left(t, s_{i, j}, y\left(s_{i, j}\right)\right)\right| \leq C_{\mathrm{Q}} \vartheta\|x-y\|
$$

for all $x, y \in S_{r_{0}}$. Thus

$$
\left\|\left(I_{\Delta} \mathbf{Q}\right)(x)-\left(I_{\Delta} \mathbf{Q}\right)(y)\right\| \leq C_{\Delta} C_{\mathbf{Q}}\left(k\left(r_{0}\right) p\left(r_{0}\right)+\left(r_{0} k\left(r_{0}\right)+F_{0}\right) \vartheta\right)\|x-y\| .
$$

Hence, the assertion follows by using the same arguments as in the proof of Theorem 4.2.

In the following theorem, the total error $x-x_{n}^{\mathrm{Q}, \Delta}$ will be estimated.

Theorem 7.3 Let the assumptions of Theorems 4.2 and 7.1 be satisfied. Then, the error $e_{n}^{\mathrm{Q}, \Delta}:=x_{n}-x_{n}^{\mathrm{Q}, \Delta}$ satisfies

$$
\left\|e_{n}^{\mathrm{Q}, \Delta}\right\| \leq \frac{1}{1-L_{\Delta}} \max _{0 \leq \ell<n} \delta_{\ell}
$$

with

$$
\delta_{\ell}:=\left\|g-I_{\Delta} g\right\|+\left\|T_{0}\left(x_{\ell}\right)-I_{\Delta} \mathbf{Q}_{0}\left(x_{\ell}\right)\right\|
$$

and $L_{\Delta}$ as in (7.3).

Proof. Recall the definition of $\mathbf{g}, \mathbf{Q}$, and $\mathbf{Q}_{0}$ as in (7.1) and the fully discrete iteration

$$
x_{n+1}^{\mathrm{Q}, \Delta}:=I_{\Delta} \mathbf{Q}\left(x_{n}^{\mathrm{Q}, \Delta}\right) .
$$

Also recall (cf. (4.1)) that the $(n+1)$ st iterate (without interpolation and quadrature) is given by

$$
x_{n+1}=g+T_{0} x_{n} .
$$

The interpolation operator $I_{\Delta}$ was defined for pairs $\left(t_{i}, f_{i}\right)_{i=0}^{N}$ and we extend the definition to continuous function by setting

$$
I_{\Delta} f:=I_{\Delta}(\mathbf{f}) \quad \text { with } \quad \mathbf{f}:=\left(f\left(t_{i}\right)\right)_{i=0}^{N} .
$$

From (7.8) we conclude the relation

$$
\begin{aligned}
e_{n+1}^{\mathrm{Q}, \Delta} & =g-I_{\Delta} g \\
& +T_{0}\left(x_{n}\right)-I_{\Delta} \mathbf{Q}_{0}\left(x_{n}\right) \\
& +I_{\Delta}\left(\mathbf{Q}_{0}\left(x_{n}\right)-\mathbf{Q}_{0}\left(x_{n}^{\mathrm{Q}, \Delta}\right)\right) .
\end{aligned}
$$

For the third summand, we get (cf. (7.6))

$$
\left\|I_{\Delta}\left(\mathbf{Q}_{0}\left(x_{n}\right)-\mathbf{Q}_{0}\left(x_{n}^{\mathrm{Q}, \Delta}\right)\right)\right\| \stackrel{(7.6)}{\leq} L_{\Delta}\left\|x_{n}-x_{n}^{\mathrm{Q}, \Delta}\right\|
$$

so that

$$
\left\|e_{n+1}^{\mathrm{Q}, \Delta}\right\| \leq \delta_{n}+L_{\Delta}\left\|e_{n}^{\mathrm{Q}, \Delta}\right\|
$$


By using $e_{0}^{\mathrm{Q}, \Delta}=0$, we get by induction

$$
\left\|e_{n}^{\mathrm{Q}, \Delta}\right\| \leq \sum_{\ell=0}^{n-1} L_{\Delta}^{\ell} \delta_{n-1-\ell} \leq \frac{1}{1-L_{\Delta}} \max _{0 \leq \ell<n} \delta_{n} .
$$

We are left with an estimate of $\delta_{n}$ and first consider the second summand in the definition of $\delta_{n}$. We split the quadrature error from the interpolation error via the estimate

$$
\left\|T_{0}\left(x_{n}\right)-I_{\Delta} \mathbf{Q}_{0}\left(x_{n}\right)\right\| \leq\left\|\left(I-I_{\Delta}\right) T_{0}\left(x_{n}\right)\right\|+C_{\Delta}\left\|\left(\mathbf{T}_{0}\left(x_{n}\right)-\mathbf{Q}_{0}\left(x_{n}\right)\right)\right\|,
$$

where $\mathbf{T}_{0}\left(x_{n}\right):=\left(\left(T_{0}\left(x_{n}\right)\right)\left(t_{i}\right)\right)_{i=0}^{N}$. These terms are small only if $x_{n}$ and $T_{0}\left(x_{n}\right)$ are (uniformly) smooth with respect to $n$. In this light, we will investigate a recursion for the derivatives of $x_{n}$. The iteration for $x_{n}^{(\ell)}, \ell \geq 1$, can be written in the form

$$
x_{n+1}^{(\ell)}=g^{(\ell)}+\left(F_{n} \mathcal{V}_{n}\right)^{(\ell)} \text {, }
$$

where (cf. (5.5))

$$
\mathcal{V}_{n}(t):=\int_{0}^{t} v\left(t, s, x_{n}(s)\right) d s \quad \text { and } \quad F_{n}:=f\left(\cdot, x_{n}(\cdot)\right)
$$

From (5.8) we conclude that

$$
x_{n+1}^{(\ell)}=g_{\ell}\left(x_{n}\right)+x_{n}^{(\ell)} T_{0}\left(\partial_{2} f, v, x_{n}\right)
$$

with

$$
\begin{aligned}
g_{\ell}(x) & :=g^{(\ell)}+q_{\ell}^{\mathrm{I}}\left(\left(H_{0}\left(\partial^{\mu} f, \partial^{\nu} v, x\right)\right)_{(\mu, \nu) \in \iota_{\ell-1}^{2,3}},\left(x^{(r)}\right)_{r=1}^{\ell-1}\right) \\
& +q_{\ell}^{\mathrm{II}}\left(\left(T_{0}\left(\partial^{\mu} f, \partial_{1}^{\nu} v, x\right)\right)_{(\mu, \nu) \in \iota_{\ell}^{2,1}},\left(x^{(r)}\right)_{r=1}^{\ell-1}\right) .
\end{aligned}
$$

Theorem 7.4 Let (3.1) and (3.2) be satisfied. Let $x_{0}=g_{0}$. Let the assumptions of Theorems 4.1, 4.2, and Theorem 5.1 be satisfied for some $m \geq 1$. Let $\beta_{0}:=r_{0}+\rho_{0}$, where $r_{0}$ is as in Theorem 4.2 and (cf. (5.3))

$$
\rho_{\ell}:=\psi_{\ell}\left(\|g\|_{\ell},\|f\|_{\ell},\|v\|_{\ell}, \frac{1}{1-|f|_{1}|v|_{0}}\right) \quad 0 \leq \ell \leq m .
$$

For $\eta>0$ and $\ell \in \mathbb{N}_{0}$, let

$$
C_{\eta}^{\ell}(I):=\left\{u \in C^{\ell}(I) \mid\|u\|_{\ell} \leq \eta\right\} .
$$

We assume that the functions $f \in C^{m}\left(I \times\left[-\beta_{0}, \beta_{0}\right]\right)$ and $v \in C^{m}\left(I \times I \times\left[-\beta_{0}, \beta_{0}\right]\right)$ satisfy

$$
\begin{gathered}
\left\|\partial^{\mu} f(t, \xi)-\partial^{\mu} f(t, \zeta)\right\| \leq C_{\partial^{\mu} f, \beta_{0}}^{\mathrm{I}}|\xi-\zeta| \quad \text { and } \quad\left\|\partial^{\mu} f(t, \xi)\right\| \leq C_{\partial^{\mu} f, \beta_{0}}^{\mathrm{II}} \\
\left\|\partial^{\nu} v(t, s, \xi)-\partial^{\nu} v(t, s, \zeta)\right\| \leq C_{\partial^{\nu} v, \beta_{0}}^{\mathrm{I}}|\xi-\zeta| \quad \text { and } \quad\left\|\partial^{\nu} v(t, s, \xi)\right\| \leq C_{\partial^{\nu} v, \beta_{0}}^{\mathrm{II}}
\end{gathered}
$$

for all $\xi, \zeta \in\left[-\beta_{0}, \beta_{0}\right], t, s \in I, \mu \in \iota_{m}^{2}$, and $\nu \in \iota_{m}^{3}$. Then, $x \in C^{m}(I)$ and the iteration (7.11) converges towards $x$ for all $0 \leq \ell \leq m$ :

$$
\left\|x^{(\ell)}-x_{n+1}^{(\ell)}\right\| \leq \tilde{C}_{\ell}(n+1) \eta_{1}^{n}
$$

for some $\left.\eta_{1} \in\right] 0,1\left[\right.$ and $\tilde{C}_{\ell}>0$. Furthermore, there are constants $\beta_{\ell}>0$ such that

$$
x_{n} \in C_{\beta_{\ell}}^{\ell}(I) \cap C_{\beta_{0}}^{0}(I) \quad \forall n \in \mathbb{N} \quad \forall 0 \leq \ell \leq m .
$$


Proof. We apply Theorem 4.2 to conclude that $\left\|x^{(\ell)}\right\| \leq \rho_{\ell}$ holds for all $0 \leq \ell \leq m$. Theorem 4.1 implies $x_{n} \in S_{r_{0}}(x) \subset S_{\beta_{0}}(x)$ so that

$$
\left\|x_{n}\right\| \leq\left\|x_{n}-x\right\|+\|x\| \leq \beta_{0}=r_{0}+\rho_{0} .
$$

Note that $T_{0}$ and $H_{0}$ are Lipschitz continuous with respect to the last argument: For functions $x, y \in C_{\beta_{0}}^{0}(I)$ it is easy to see that

$$
\begin{gathered}
\left\|T_{0}\left(\partial^{\mu} f, \partial_{1}^{j} v, x\right)-T_{0}\left(\partial^{\mu} f, \partial_{1}^{j} v, y\right)\right\| \leq C_{\partial^{\mu} f, \partial_{1}^{j} v, \beta_{0}}^{\mathrm{I}}\|x-y\|, \\
\left\|H_{0}\left(\partial^{\lambda} f, \partial^{\nu} v, x\right)-H_{0}\left(\partial^{\lambda} f, \partial^{\nu} v, y\right)\right\| \leq C_{\partial^{\lambda} f, \partial^{\nu} v, \beta_{0}}^{\mathrm{I}}\|x-y\|,
\end{gathered}
$$

and

$$
\begin{aligned}
& \left\|T_{0}\left(\partial^{\mu} f, \partial_{1}^{j} v, x\right)\right\| \leq C_{\partial^{\mu} f, \partial_{1}^{j} v, \beta_{0}}^{\mathrm{II}}, \\
& \left\|H_{0}\left(\partial^{\lambda} f, \partial^{\nu} v, x\right)\right\| \leq C_{\partial^{\lambda} f, \partial^{\nu} v, \beta_{0}}^{\mathrm{II}}
\end{aligned}
$$

for all $(\mu, j) \in \iota_{m}^{2,1}$ and $(\lambda, \nu) \in \iota_{m-1}^{2,3}$ with (cf. (7.13))

$$
C_{h, w, \beta_{0}}^{\mathrm{I}}:=C_{h, \beta_{0}}^{\mathrm{I}} C_{w, \beta_{0}}^{\mathrm{II}}+C_{h, \beta_{0}}^{\mathrm{II}}, C_{w, \beta_{0}}^{\mathrm{I}} \quad \text { and } \quad C_{h, w, \beta_{0}}^{\mathrm{II}}:=C_{h, \beta_{0}}^{\mathrm{II}} C_{w, \beta_{0}}^{\mathrm{II}} .
$$

For $1 \leq \ell \leq m$, let

$$
\mathbf{H}_{\ell}(x)=\left(H_{0}\left(\partial^{\mu} f, \partial^{\nu} v, x\right)\right)_{(\mu, \nu) \in \iota_{\ell-1}^{2,3}} \quad \text { and } \quad \mathbf{T}_{\ell}(x)=\left(T_{0}\left(\partial^{\mu} f, \partial_{1}^{\nu} v, x\right)\right)_{(\mu, \nu) \in \iota_{\ell}^{2,1}}
$$

For $x, y \in C_{\beta_{0}}^{0}(I)$ and $1 \leq \ell \leq m$, we conclude from (7.14) that

$$
\begin{aligned}
\left\|\mathbf{H}_{\ell}(x)-\mathbf{H}_{\ell}(y)\right\| & \leq C_{\mathbf{H}, \ell-1, \beta_{0}}^{\mathrm{I}}\|x-y\| \quad \text { and } \quad\left\|\mathbf{H}_{\ell}(x)\right\| \leq C_{\mathbf{H}, \ell-1, \beta_{0}}^{\mathrm{II}}, \\
\left\|\mathbf{T}_{\ell}(x)-\mathbf{T}_{\ell}(y)\right\| & \leq C_{\mathbf{T}, \ell, \beta_{0}}^{\mathrm{I}}\|x-y\| \quad \text { and } \quad\left\|\mathbf{T}_{\ell}(x)\right\| \leq C_{\mathbf{T}, \ell, \beta_{0}}^{\mathrm{II}}
\end{aligned}
$$

holds with

$$
C_{\mathbf{H}, \ell-1, \beta_{0}}^{\mathrm{IIII}}:=\max _{(\mu, \nu) \in \iota_{\ell-1}^{2,3}} C_{\partial^{\mu} f, \partial^{\nu} v, \beta_{0}}^{\mathrm{I}, \mathrm{II}} \quad \text { and } \quad C_{\mathbf{T}, \ell, \beta_{0}}^{\mathrm{IIII}}:=\max _{(\mu, \nu) \in \iota_{\ell}^{2,1}} C_{\partial^{\mu} f, \partial^{\nu} v, \beta_{0}}^{\mathrm{I}, \mathrm{II}} .
$$

Let $\mathbf{x}=\left(x_{r}\right)_{r=1}^{\ell-1} \in \mathbb{R}^{\ell-1}$ with $\|\mathbf{x}\|_{\max }<\beta$ for $\beta>0$. Since $q_{\ell}^{\mathrm{I}, \mathrm{II}}$ in (5.8), (7.12) are polynomials, there exists constants $C_{\ell, \beta}^{\mathrm{I}}, C_{\ell, \beta}^{\mathrm{II}}$ depending only on $\beta$ such that

$$
\begin{aligned}
\left|q_{\ell}^{\mathrm{I}}(\boldsymbol{\zeta}, \mathbf{x})-q_{\ell}^{\mathrm{I}}(\boldsymbol{\xi}, \mathbf{x})\right| \leq C_{\ell, \beta}^{\mathrm{I}}\|\boldsymbol{\zeta}-\boldsymbol{\xi}\|_{\max } & \forall \boldsymbol{\zeta}=\left(\zeta_{\mu, \nu}\right)_{(\mu, \nu) \in \iota_{\ell-1}^{2,3}}, \boldsymbol{\xi}=\left(\xi_{\mu, \nu}\right)_{(\mu, \nu) \in \iota_{\ell-1}^{2,3}} \\
\left|q_{\ell}^{\mathrm{II}}(\boldsymbol{\zeta}, \mathbf{x})-q_{\ell}^{\mathrm{II}}(\boldsymbol{\xi}, \mathbf{x})\right| \leq C_{\ell, \beta}^{\mathrm{II}}\|\boldsymbol{\zeta}-\boldsymbol{\xi}\|_{\max } & \forall \boldsymbol{\zeta}=\left(\zeta_{\mu, \nu}\right)_{(\mu, \nu) \in \iota_{\ell-1}^{2,3}}, \boldsymbol{\xi}=\left(\xi_{\mu, \nu}\right)_{(\mu, \nu) \in \iota_{\ell-1}^{2,3}} .
\end{aligned}
$$

Hence, for $x, y \in C_{\beta}^{\ell}(I) \cap C_{\beta_{0}}^{0}(I)$ it holds

$$
\begin{aligned}
\left\|g_{\ell}(x)-g_{\ell}(y)\right\| & \leq C_{\ell, \beta}^{\mathrm{I}}\left\|\mathbf{H}_{\ell}(x)-\mathbf{H}_{\ell}(y)\right\|+C_{\ell, \beta}^{\mathrm{II}}\left\|\mathbf{T}_{\ell}(x)-\mathbf{T}_{\ell}(y)\right\| \\
& \leq C_{\mathbf{H}, \mathbf{T}, \ell, \beta, \beta_{0}}\|x-y\| \quad \text { with } \quad C_{\mathbf{H}, \mathbf{T}, \ell, \beta, \beta_{0}}:=C_{\ell, \beta}^{\mathrm{I}} C_{\mathbf{H}, \ell-1, \beta_{0}}^{\mathrm{I}}+C_{\ell, \beta}^{\mathrm{II}} C_{\mathbf{T}, \ell, \beta_{0}}^{\mathrm{I}} .
\end{aligned}
$$

Next, we consider the convergence of the recursion (7.11). It holds

$$
\left\|x^{(\ell)}-x_{n+1}^{(\ell)}\right\| \leq\left\|g_{\ell}(x)-g_{\ell}\left(x_{n}\right)\right\|+\rho_{\ell}\left\|T_{0}\left(\partial_{2} f, v, x\right)-T_{0}\left(\partial_{2} f, v, x_{n}\right)\right\|+\left\|x^{(\ell)}-x_{n}^{(\ell)}\right\|\left\|T_{0}\left(\partial_{2} f, v, x_{n}\right)\right\| .
$$


Since $x, x_{n} \in C_{\beta_{0}}^{0}(I)$ we conclude that

$$
\left\|T_{0}\left(\partial_{2} f, v, x\right)-T_{0}\left(\partial_{2} f, v, x_{n}\right)\right\| \leq C_{\partial_{2} f, v, \beta_{0}}^{\mathrm{I}}\left\|x-x_{n}\right\| \quad \text { and } \quad\left\|T_{0}\left(\partial_{2} f, v, x_{n}\right)\right\| \leq C_{\partial_{2} f, v, \beta_{0}}^{\mathrm{II}}
$$

holds and (cf. (4.7))

$$
\left\|x^{(\ell)}-x_{n+1}^{(\ell)}\right\| \leq \gamma_{\ell} L^{n}+\eta_{0}\left\|x^{(\ell)}-x_{n}^{(\ell)}\right\|
$$

with

$\eta_{0}:=C_{\partial_{2} f, v, \beta, \beta_{0}}^{\mathrm{II}}:=\left\|\partial_{2} f\right\|\|v\| \stackrel{(5.1)}{<} 1, \quad \gamma_{\ell}:=2 r_{0} \frac{C_{\mathbf{H}, \mathbf{T}, \ell, \beta, \beta_{0}}+\rho_{\ell} C_{\partial_{2} f, v, \beta_{0}}^{\mathrm{I}}}{1-L}, \quad \beta:=\|x\|_{\ell-1}+\sup _{n \in \mathbb{N}}\left\|x_{n}\right\|_{\ell-1}$.

By induction we obtain from (7.15) the convergence of $x_{n+1}^{(\ell)}$ towards $x^{(\ell)}$ :

$$
\left\|x^{(\ell)}-x_{n+1}^{(\ell)}\right\| \leq \gamma_{\ell} \sum_{k=0}^{n} \eta_{0}^{k} L^{n-k}+\eta_{0}^{n+1}\left\|x^{(\ell)}-x_{0}^{(\ell)}\right\| .
$$

Let $\left.\eta_{1}:=\max \left\{\eta_{0}, L\right\} \in\right] 0,1\left[\right.$ and observe $\left\|x^{(\ell)}-x_{0}^{(\ell)}\right\| \leq \rho_{\ell}+\left\|g^{(\ell)}\right\|$. Then,

$$
\left\|x^{(\ell)}-x_{n+1}^{(\ell)}\right\| \leq \gamma_{\ell} C_{\ell}(n+1) \eta_{1}^{n} \quad \text { with } \quad C_{\ell}=\gamma_{\ell}+\eta_{0}\left(\rho_{\ell}+\left\|g^{(\ell)}\right\|\right) .
$$

In addition it holds

$$
\sup _{n \geq 0}\left\|x_{n}^{(\ell)}\right\| \leq \rho_{\ell}+\gamma_{\ell} C_{\ell} \sup _{n \geq 0}\left((n+1) \eta_{1}^{n}\right)
$$

In order to estimate the quantities $\delta_{\ell}$ in (7.7) we have to impose some concrete assumption on the quadrature method $Q_{i}$. We assume that

$$
\max _{1 \leq i \leq N}\left|\int_{0}^{t_{i}} v\left(t_{i}, \tau, x_{n}(\tau)\right) d \tau-Q_{i}\left(x_{n}\right)\right| \leq C h^{\ell} \nu_{\ell}
$$

holds for $0 \leq \ell \leq \ell_{0}$, where $\ell_{0}$ depends on the exactness degree of the quadrature $Q_{t}$ and the smoothness of $v$ and $x$ :

$$
\nu_{\ell}:=\max _{0 \leq i \leq N}\left\|V_{i}^{(\ell)}\right\| \quad \text { with } \quad V_{i}(s):=v\left(t_{i}, s, x(s)\right) .
$$

Theorem 7.5 Let the assumptions of Theorems 7.3, 7.4, and A.1 be satisfied. Then, for $1 \leq \ell \leq \min \{4, m\}$ with $m$ as in Theorem $\% .4$, it holds

$$
\left\|e_{n}^{\mathrm{Q}, \Delta}\right\| \leq \frac{C h^{\ell}}{1-L_{\Delta}}\left(\left\|g^{(\ell)}\right\|+\rho_{\ell}\|v\|_{\ell}\|f\|_{\ell}\right) \text {. }
$$

Proof. Note that $x_{n} \in C_{\beta_{\ell}}^{\ell}(I) \cap C_{\beta_{0}}^{0}(I)$ for all $0 \leq \ell \leq \min \left\{m, \ell_{0}\right\}$ (cf. (7.16)) and

$$
\left\|\left(\mathbf{T}_{0}\left(x_{n}\right)-\mathbf{Q}_{0}\left(x_{n}\right)\right)\right\| \leq C_{f, \beta_{0}}^{\mathrm{II}} \max _{0 \leq i \leq N}\left|\int_{0}^{t_{i}} v\left(t_{i}, \tau, x_{n}(\tau)\right) d \tau-Q_{i}\left(x_{n}\right)\right| \stackrel{(7.16)}{\leq} C_{f, \beta_{0}}^{\mathrm{II}} C h^{\ell} \nu_{\ell} .
$$

Similarly as for the considerations $(5.7)$ related to $F^{(\ell)}$ in the proof of Theorem 5.1 we conclude that

$$
\left\|V_{i}^{(\ell)}\right\| \leq C_{\ell}\|x\|_{\ell}\|v\|_{\ell} \leq C_{\ell} \rho_{\ell}\|v\|_{\ell}
$$


holds so that

$$
\left\|\left(\mathbf{T}_{0}\left(x_{n}\right)-\mathbf{Q}_{0}\left(x_{n}\right)\right)\right\| \leq C_{f, \beta_{0}}^{\mathrm{II}}\|v\|_{\ell} C_{\ell} \rho_{\ell} C h^{\ell} .
$$

Since $g \in C^{\ell}$ for $0 \leq \ell \leq m \leq 4$ we can apply (A.1) to get

$$
\left\|g-I_{\Delta} g\right\| \leq C h^{\ell}\left\|g^{(\ell)}\right\| \text {. }
$$

Finally, we observe that

$$
\left\|\left(I-I_{\Delta}\right) T_{0}\left(x_{n}\right)\right\| \leq C h^{\ell}\left\|\left(T_{0} x_{n}\right)^{(\ell)}\right\| .
$$

Since $T_{0}\left(x_{n}\right)=F_{n} \mathcal{V}_{n}(\operatorname{cf}(7.10))$, we conclude from (5.9) that

$$
\left\|\left(T_{0} x_{n}\right)^{(\ell)}\right\| \leq C \rho_{\ell}\|f\|_{\ell}\|v\|_{\ell} .
$$

\section{Adaptive Techniques for Quadrature and Spline Col- location}

In this section, we will introduce an adaptive strategy for the iteration (6.4). Such an approach is preferable compared to the use of uniform meshes if the solution $x(t)$ has large derivatives only in a local part of the interval such as $t^{\alpha}$ for non-integer or large $\alpha$. In such cases, non-uniformly spaced quadrature and interpolation points can be much more efficient.

To set up an (heuristic) strategy in order to balance and to estimate the different errors we recall that the total error consists of the following four terms:

$$
\begin{array}{ll}
E_{n}^{\mathrm{it}}:=\frac{L^{n}}{1-L}\left\|x_{1}^{\mathrm{Q}, \Delta}-x_{0}^{\mathrm{Q}, \Delta}\right\| & (\mathrm{cf} .(7.4)), \\
E_{\Delta}^{\text {data }}:=\frac{1}{1-L_{\Delta}}\left\|g-I_{\Delta} g\right\| & (\text { cf. }(7.7)), \\
E_{\Delta, n}^{\text {int }}:=\frac{1}{1-L_{\Delta}}\left\|\left(I-I_{\Delta}\right) T_{0}\left(x_{n}\right)\right\| & (\text { cf. }(7.7) /(7.9)), \\
E_{n}^{\text {quad }}:=\frac{C_{\Delta}}{1-L_{\Delta}}\left\|\left(\mathbf{T}_{0}\left(x_{n}\right)-\mathbf{Q}_{0}\left(x_{n}\right)\right)\right\| & (\text { cf. }(7.7) /(7.9)) .
\end{array}
$$

Let $\varepsilon>0$ denote a given tolerance. Our goal is that each of these four error terms is of order $\varepsilon / 4$. After $x_{1}^{\mathrm{Q}, \Delta}$ is computed, the total number $n$ of iterations is determined by the condition

$$
\frac{L^{n}}{1-L}\left\|x_{1}^{\mathrm{Q}, \Delta}-x_{0}^{\mathrm{Q}, \Delta}\right\| \leq \varepsilon / 4
$$

The initial mesh $\Delta$, with, possibly, variable mesh widths, is chosen such that $E_{\Delta}^{\text {data }} \leq \varepsilon / 4$. A very simple adaptive procedure for this purpose is given by starting with a coarse mesh $\mathcal{G}_{0}$ and determining the quantities ${ }^{2}$

$$
\forall \tau \in \mathcal{G}_{0} \quad \lambda_{\tau}:=\frac{1}{1-L_{\Delta}}\left\|g-I_{\Delta} g\right\|_{C^{0}(\tau)} \quad \text { and } \quad \lambda_{\max }:=\max \lambda_{\tau}
$$

\footnotetext{
${ }^{2}$ Note that in compuations the evaluation of $\left\|g-I_{\Delta} g\right\|_{C^{0}(\tau)}$, typically, has to be replaced by the maximum over $\left|\left(g-I_{\Delta} g\right)\left(\left(t_{\tau, i}\right)\right)\right|$ for a finite set $\left\{t_{\tau, i}: 1 \leq i \leq n_{\tau}\right\} \subset \tau$.
} 
Then those intervals $\tau$ are bisected which satisfy $\lambda_{\tau} \geq \alpha \lambda_{\max }$ for some given threshold $\alpha \in$ ] 0,1 [. The resulting mesh is denoted by $\mathcal{G}_{1}$ and this refinement step is repeated until $\lambda_{\max } \leq$ $\varepsilon / 4$. This gives the starting mesh $\Delta_{0}$ for the iteration (6.4).

Since the accuracy of the quadrature method is related to the smoothness of both functions, $v$ and $x_{n}^{\mathrm{Q}, \Delta}$, we will base the mesh adaptation on an error indicator for the local quadrature: If a new quadrature point is added to the mesh by this indicator, then, the spline space for the next iteration will be enriched as well by this quadrature point as a new collocation point.

In order to get an indication on the size of $E_{\Delta, n}^{\text {int }}$ we evaluate the new iterate $x_{n+1}^{\mathrm{Q}, \Delta}$ at the midpoints of the intervals $m_{i}:=\frac{1}{2}\left(t_{i}+t_{i-1}\right)$ and compare these values with

$$
x_{n+1}^{\mathrm{Q}}\left(m_{i}\right):=g\left(m_{i}\right)+f\left(m_{i}, x_{n}^{\mathrm{Q}, \Delta}\left(m_{i}\right)\right) Q_{m_{i}}\left(x_{n}^{\mathrm{Q}, \Delta}\right) .
$$

Then we use

$$
\frac{1}{1-L_{\Delta}} \max _{1 \leq i \leq N}\left|x_{n+1}^{\mathrm{Q}}\left(m_{i}\right)-x_{n+1}^{\mathrm{Q}, \Delta}\left(m_{i}\right)\right|
$$

as an indicator for the quantity $E_{\Delta, n}^{\text {int }}$. The following section is concerned with the quadrature error $E_{n}^{\text {quad }}$.

\subsection{Adaptive Quadrature}

An adaptive quadrature method has been introduced, e.g., in [17] and we briefly sketch the procedure which takes into account that, locally, the variations of the function could require a higher resolution by the nodal points while in other parts a lower resolution might be sufficient. The proposed method is based on Simpson's rule. Simpson's rule on an interval $\tau=(a, b)$ is given by

$$
S_{\tau}(f)=\frac{h}{3}\left(f(a)+4 f\left(m_{\tau}\right)+f(b)\right),
$$

where $m_{\tau}=\frac{1}{2}(a+b)$ is the midpoint of $\tau$. It is well known that, for $f \in C^{4}(\tau)$, there exists a value $d_{1} \in \tau$ so that:

$$
I_{\tau}(f):=\int_{a}^{b} f(x) d x=S_{\tau}(f)-h^{5} \frac{f^{(4)}\left(d_{1}\right)}{90} .
$$

\section{Refinement}

A composite Simpson rule for $\tau$ is given by splitting $\tau=\tau^{\mathrm{I}} \cup \tau^{\mathrm{II}}$ with $\tau^{\mathrm{I}}=\left(a, m_{\tau}\right)$ and $\tau^{\mathrm{II}}=\left(m_{\tau}, b\right)$

$$
I_{\tau}(f) \approx S_{\tau^{\mathrm{I}}}(f)+S_{\tau^{\mathrm{II}}}(f)=\frac{h}{6}\left(f(a)+4 f\left(m_{\tau^{\mathrm{I}}}\right)+2 f\left(m_{\tau}\right)+4 f\left(m_{\tau^{\mathrm{II}}}\right)+f(b)\right) .
$$

Note that only two additional function evaluations (compared to (8.2)) are required. In formula (8.4) the step size is $h / 2$, which accounts for the factors $h / 6$ on the right-hand side of the equation. Furthermore, if $f \in C^{4}(\tau)$, there exists some value $d_{2} \in \tau$ so that:

$$
\begin{aligned}
I_{\tau}(f) & =S_{\tau^{\mathrm{I}}}(f)+S_{\tau^{\mathrm{II}}}(f)-\frac{h^{5}}{2^{5}} \frac{f^{(4)}\left(d_{2^{\mathrm{I}}}\right)}{90}-\frac{h^{5}}{2^{5}} \frac{f^{(4)}\left(d_{2^{\mathrm{II}}}\right)}{90} \\
& =S_{\tau^{\mathrm{I}}}(f)+S_{\tau^{\mathrm{II}}}(f)-\frac{h^{5}}{16} \frac{f^{(4)}\left(d_{2}\right)}{90} .
\end{aligned}
$$


Assume that $f^{(4)}\left(d_{1}\right) \approx f^{(4)}\left(d_{2}\right)$. Then, the right-hand sides of equations (8.3) and (8.5) are employed to obtain the relation

$$
S_{\tau}(f)-h^{5} \frac{f^{(4)}\left(d_{2}\right)}{90} \approx S_{\tau^{\mathrm{I}}}(f)+S_{\tau^{\mathrm{II}}}(f)-\frac{h^{5}}{16} \frac{f^{(4)}\left(d_{2}\right)}{90}
$$

which can be written as

$$
-h^{5} \frac{f^{(4)}\left(d_{2}\right)}{90} \approx \frac{16}{15}\left(S_{\tau^{\mathrm{I}}}(f)+S_{\tau^{\mathrm{II}}}(f)-S_{\tau}(f)\right) .
$$

Then (8.7) is substituted into (8.5) to obtain the error indicator:

$$
\left|I_{\tau}(f)-\left(S_{\tau^{\mathrm{I}}}(f)+S_{\tau^{\mathrm{II}}}(f)\right)\right| \approx \frac{1}{15}\left|S_{\tau^{\mathrm{I}}}(f)+S_{\tau^{\mathrm{II}}}(f)-S_{\tau}(f)\right| .
$$

This justifies the following test.

\section{Accuracy Test}

Assume that the tolerance $\varepsilon_{\tau}>0$ is specified for the interval $\tau=[a, b]$. If

$$
\frac{1}{15}\left|S_{\tau^{\mathrm{I}}}(f)+S_{\tau^{\mathrm{II}}}(f)-S_{\tau}(f)\right|<\varepsilon_{\tau},
$$

we infer that

$$
\left|I_{\tau}(f)-\left(S_{\tau^{\mathrm{I}}}(f)+S_{\tau^{\mathrm{II}}}(f)\right)\right| \lesssim \varepsilon_{\tau} .
$$

Thus the composite Simpson rule (8.4) is used to approximate the integral

$$
I_{\tau}(f) \approx\left(S_{\tau^{\mathrm{I}}}(f)+S_{\tau^{\mathrm{II}}}(f)\right)
$$

and we use the computable value of $\varepsilon_{\tau}$ (cf. (8.9)) as an error indicator.

The adaptive quadrature is based on Simpson's rules (8.2) and (8.4) and controlled by the accuracy test (8.9): Assume that a tolerance $\varepsilon>0$ is given for approximation the integral $\int_{0}^{1} f$. For $0 \leq \ell \leq \ell_{\max }$, we will generate recursively a sequence $\mathcal{G}_{\ell}=\left\{\tau_{\ell, i}: 1 \leq i \leq N_{\ell}\right\}$ of sets of disjoint subintervals in $[0,1]$. We assume that $\mathcal{G}_{0}$ is a coarse (typically uniform) partition of $[0,1]$ and the approximate integral value is initialized by setting $S:=0$. At level $\ell=0,1, \ldots$, we compute for each interval $\tau_{\ell, i} \in \mathcal{G}_{\ell}$ the quantity

$$
\delta_{\ell, i}:=\frac{1}{15}\left|S_{\tau_{\ell, i}^{\mathrm{I}}}(f)+S_{\tau_{\ell, i}^{\mathrm{II}}}(f)-S_{\tau_{\ell, i}}(f)\right| .
$$

If $\delta_{\ell, i} \leq \frac{\varepsilon}{\sharp \mathcal{G}_{\ell}}$ then $S \leftarrow S+S_{\tau_{\ell, i}^{\mathrm{I}}}(f)+S_{\tau_{\ell, i}^{\mathrm{II}}}(f)$. Otherwise, we bisect the intervals $\tau_{\ell, i}$ and set $\mathcal{G}_{\ell+1} \leftarrow\left(\mathcal{G}_{\ell+1} \backslash\left\{\tau_{\ell, i}\right\}\right) \cup\left\{\tau_{\ell, i}^{\mathrm{I}}, \tau_{\ell, i}^{\mathrm{II}}\right\}$. The algorithm is terminated if no subinterval is refined furthermore.

Now, in each fixed point iteration we use this method to approximate the integrals

$$
\int_{0}^{t_{i}} v\left(t_{i}, s, x_{n}^{\mathrm{Q}, \Delta}(s)\right) d s, \quad i=1, \ldots, N
$$

with an accuracy of order $\varepsilon / 4$. 


\section{Numerical Examples}

In this section, some examples are presented to test the accuracy and application of the proposed method.

Example 9.1 Consider the following quadratic Volterra integral equation with the exact solution $x(t)=\frac{t^{10}}{10}$ :

$$
x(t)=g(t)+f(t, x(t)) \int_{0}^{t} v(t, s, x(s)) d s, \quad t, s \in[0,1],
$$

where

$$
\begin{aligned}
g(t) & :=t^{10} / 10-t /(1+t) \ln \left(1+t^{10} / 10\right)\left(t^{3} / 2+t^{11} / 110\right), \\
f(t, x) & :=(t /(1+t)) \ln (1+x) \\
v(t, s, x) & :=t s+x
\end{aligned}
$$

The function $g$ is nondecreasing and nonnegative on $I$ and $g_{0}=0,\|g\|=0.0757$. The function $f$ is nonnegative and nondecreasing with respect to both variables on $I$. Further, we have $\partial_{2} f(t, x)=(t /(1+t)) /(1+x)$ so that $k(r)=\sup \left\{\partial_{2} f(t, x):(t, x) \in I \times J\right\}=1 / 2$ for any $r>0$. Moreover, the function $t \rightarrow v(t, s, x)$ is nondecreasing on $I$ and we have $v(t, s, x) \leq 1+x$ for $t, s \in I$ and $x \geq 0$, thus $p(x)=1+x$. Further, $v(t, s, x)$ satisfies the Lipschitz condition with respect to variable $x$ with Lipschitz constant $\vartheta=1$. Finally, consider the inequalities from the Assumption 3.4(vi) (cf. Theorem 3.5) and assumption (b) in Theorem 4.2 which have the forms

$$
\begin{array}{r}
0.0757+\frac{1}{2} r(1+r) \leq r, \\
\frac{1}{2}(1+r)+\frac{1}{2} r<1 .
\end{array}
$$

It is easy to check that $r_{0}=0.1862$ is the minimal solution of this system of inequalities. Then the integral equation has a unique continuous, nonnegative and nondecreasing solution on $I=[0,1]$ for which $x \in[0,0.1862]$. We see that our theory gives us a range with is close to the exact range $[0,0.1]$ of the exact solution $x(t)$. To solve the equation by the fixed point iteration, we need to determine the iteration number $n$ and the number of mesh points $N$ by (8.1(1)) and (8.1(3)). Note that $R_{0}=r_{0} k\left(r_{0}\right)+F_{0}=0.0931$ and $L=k\left(r_{0}\right) p\left(r_{0}\right)+R_{0} \vartheta=0.6862$. To simplify the consideration we use techniques as in [10] to derive a grading function $\Gamma(t)=t^{\frac{7}{3}}$ which determines the collocation points by

$$
t_{i}=\left(\frac{i}{N}\right)^{\frac{3}{7}}, \quad i=0,1, \ldots, N
$$

By (9.1), the number of grid points depending on the given error (8.1(3)) is obtained. Table (1) shows the results. As the collocation points we choose

$$
t_{i}=1-\left(\frac{N-i}{N}\right)^{2}, \quad i=0,1, \ldots, N
$$


Table (1) shows that, in this case, the number of collocation points is less than for the other cases.

$\varepsilon:=$ Tolerance

$N_{1}:=$ Number of collocation points with uniform mesh

$N_{2}:=$ Number of collocation points with nonuniform mesh (9.1)

$N_{3}:=$ Number of collocation points with nonuniform mesh (9.2)

$E_{1}:=$ Error in collocation points

$E_{2}:=$ Error on whole interval $I$

$I T:=$ Number of iteration

Table 1: Results for example (9.1)

\begin{tabular}{|c||c|c|c|c|c|c|c|}
\hline$\varepsilon$ & $N_{1}$ & $N_{2}$ & $N_{3}$ & $E_{1}$ & $E_{2}$ & IT & \\
\hline $10^{-7}$ & 110 & 74 & 45 & $10^{-9}$ & $10^{-8}$ & 39 & \\
$10^{-9}$ & 350 & 234 & 130 & $10^{-11}$ & $10^{-10}$ & 51 & \\
$10^{-11}$ & 850 & 739 & 400 & $10^{-13}$ & $10^{-12}$ & 63 & \\
$10^{-12}$ & 1500 & 1314 & 700 & $10^{-14}$ & $10^{-13}$ & 69 & \\
\hline
\end{tabular}

\section{A Stability and Convergence of Cubic Spline Interpo- lation}

Theorem A.1 For an interval partitioning

$$
0=t_{0}<t_{1}<\ldots<t_{N}=1, \quad \tau_{i}:=\left[t_{i-1}, t_{i}\right], \quad h_{\tau_{i}}:=t_{i}-t_{i-1}
$$

with corresponding function values $\mathbf{f}:=\left(f_{i}\right)_{i=0}^{N}$, let $u_{\Delta}$ denote the cubic interpolating spline with boundary conditions

$$
u_{\Delta}^{\prime}(0)=\frac{f_{2}-f_{0}}{t_{2}-t_{0}} \quad \text { and } \quad u_{\Delta}^{\prime}(1)=\frac{f_{N}-f_{N-2}}{t_{N}-t_{N-2}} .
$$

a. Then,

$$
\left\|u_{\Delta}\right\|_{C_{0}(I)} \leq C\|\mathbf{f}\|_{\max },
$$

where $C$ depends on the global quasi-uniformity $h / h_{\min }$ with $h_{\min }:=\min \left\{h_{\tau}: \tau \in \Delta\right\}$. Let $f \in C^{\ell}(I)$ for some $0 \leq \ell \leq 4$. Then

$$
\left\|f-u_{\Delta}\right\| \leq C h^{\ell}\left\|f^{(\ell)}\right\| .
$$

b. Let the constant of local quasi-uniformity be given by

$$
\kappa:=\max _{1 \leq i \leq N-1}\left\{\max \left\{\frac{h_{\tau_{i}}}{h_{\tau_{i+1}}}, \frac{h_{\tau_{i+1}}}{h_{\tau_{i}}}\right\}\right\}
$$


and assume $\kappa^{2}<2$. Then,

$$
\left\|u_{\Delta}\right\|_{C^{0}(I)} \leq C_{\Delta}\|\mathbf{f}\|_{\max } \quad \text { with } \quad C_{\Delta} \leq \frac{C}{2-\kappa^{2}}
$$

where $C$ only depends on $\kappa$. Let $f \in C^{\ell}(I)$ for some $0 \leq \ell \leq 4$. Then

$$
\left\|f-u_{\Delta}\right\| \leq \frac{C}{2-\kappa^{2}} h^{\ell}\left\|f^{(\ell)}\right\|
$$

Proof. For $k, p \in \mathbb{N}_{0}$, let

$$
S^{k, p}:=\left\{v \in C^{k}(I)\left|\forall \tau_{i} \quad v\right|_{\tau_{i}} \in \mathbb{P}_{p}\right\} \quad \text { and } \quad S_{0}^{k, p}:=\left\{v \in S^{k, p}: v(0)=v(1)=0\right\} .
$$

For simplicity we assume that $N \geq 4$. We construct a function $u_{\text {aux }} \in S^{4,9}$ such that

$$
u_{\mathrm{aux}}^{(k)}\left(t_{i}\right)=f_{i, k} \quad 0 \leq k \leq 4 \quad 0 \leq i \leq N,
$$

where, for $0 \leq i \leq N$ and $0 \leq k \leq 4$, the values $f_{i, k}$ are divided differences

$$
f_{i, k}:= \begin{cases}{\left[t_{j-k / 2}, \ldots, t_{j+k / 2}\right] f} & k \text { even } \\ \left.t_{j-\frac{k+1}{2}}, \ldots, t_{j-1}, t_{j+1}, \ldots, t_{j+\frac{k+1}{2}}\right] f & k \text { odd }\end{cases}
$$

which are centered at

$$
j:=j_{i, k}:= \begin{cases}\left\lceil\frac{k}{2}\right\rceil & i<\left\lceil\frac{k}{2}\right\rceil \\ N-\left\lceil\frac{k}{2}\right\rceil & i>N-\left\lceil\frac{k}{2}\right\rceil \\ i & \text { otherwise. }\end{cases}
$$

As usual for Hermite interpolation we initialize the divided differences by

$$
[\underbrace{t_{\ell}, \ldots, t_{\ell}}_{(k+1) \times}] f:=f_{\ell, k} \quad \text { for } \ell=i-1, i \text { and } 0 \leq k \leq 4
$$

and employ for the remaining differences the recursion

$$
[\underbrace{t_{i-1}, \ldots t_{i-1}}_{m \text {-times }}, \underbrace{t_{i}, \ldots, t_{i}}_{n \text {-times }}] f:=\frac{[\underbrace{t_{i-1}, \ldots t_{i-1}}_{(m-1) \text {-times }}, \underbrace{t_{i}, \ldots, t_{i}}_{n \text {-times }}] f-[\underbrace{t_{i-1}, \ldots t_{i-1}}_{m \text {-times }}, \underbrace{t_{i}, \ldots, t_{i}}_{(n-1) \text {-times }}] f}{h_{i}}
$$

for $m \geq 1$ and $n \geq 1$. On an interval $\tau=\left[t_{i-1}, t_{i}\right]$, this leads to

$$
\left.u_{\mathrm{aux}}\right|_{\tau_{i}}=\sum_{k=0}^{4} f_{i-1, k} \omega_{i-1, k}+\sum_{k=0}^{4}\left([\underbrace{t_{i-1}, \ldots, t_{i-1}}_{5 \times}, \underbrace{t_{i}, \ldots, t_{i}}_{(k+1) \times}] f\right) \omega_{i, k},
$$

where, for $0 \leq k \leq 4$,

$$
\omega_{\ell, k}(t):= \begin{cases}\left(t-t_{i-1}\right)^{k} & \ell=i-1 \\ \left(t-t_{i-1}\right)^{5}\left(t-t_{i}\right)^{k} & \ell=i\end{cases}
$$


Thus, we get for $0 \leq m \leq 4$

$$
\left|\omega_{\ell, k}^{(m)}(t)\right| \leq C_{1} \begin{cases}0 & m>k \wedge \ell=i-1 \\ h_{\tau_{i}}^{k-m} & m \leq k \wedge \ell=i-1 \\ h_{\tau_{i}}^{5+k-m} & \ell=i\end{cases}
$$

We introduce the index neighborhood (cf. (A.6))

$$
\iota_{i}:=\left\{j_{i-1,4}-2, \ldots, j_{i-1,4}+2\right\} \cup\left\{j_{i, 4}-2, \ldots, j_{i, 4}+2\right\}
$$

which contains those indices which are involved in the definition of $[\underbrace{t_{i-1}, \ldots t_{i-1}}_{m \text {-times }}, \underbrace{t_{i}, \ldots, t_{i}}_{n \text {-times }}] f$ for $0 \leq m \leq 4,0 \leq n \leq 4$ with $(m, n) \neq(0,0)$. We set

$$
h_{i, \min }:=\min \left\{h_{n}: n \in \iota_{i}\right\}
$$

and obtain

$$
[\underbrace{t_{i-1}, \ldots t_{i-1}}_{m \text {-times }}, \underbrace{t_{i}, \ldots, t_{i}}_{n \text {-times }}] f \leq C_{2} \frac{\left\|\left(f_{n, k}\right)_{n \in \iota_{i}}\right\|_{\max }}{h_{i, \min }^{m+n-k-1}} \quad \text { for } 0 \leq k \leq m+n-1 \leq 4 .
$$

Thus, for $0 \leq r \leq m \leq 4$, it holds

$$
\begin{aligned}
\left\|u_{\text {aux }}^{(m)}\right\|_{C^{0}\left(\tau_{i}\right)} \leq & \sum_{s=m}^{4} \frac{\left\|\left(f_{n, r}\right)_{n \in \iota_{i}}\right\|}{h_{i, \min }^{s-r}} h_{\tau_{i}}^{s-m}+\sum_{s=0}^{4} \frac{\left\|\left(f_{n, r}\right)_{n \in \iota_{i}}\right\|_{\tau_{i, \min }}}{h_{\tau_{i}}^{s+5-5-m},} \\
\leq & C_{1} C_{2}\left(\sum_{s=m}^{4}\left\|\left(f_{n, r}\right)_{n \in \iota_{i}}\right\|_{\max }\left(\frac{h_{\tau_{i}}}{h_{i, \min }}\right)^{s-r} h_{\tau_{i}}^{r-m}\right. \\
& \left.+\sum_{s=0}^{4}\left\|\left(f_{n, r}\right)_{n \in \iota_{i}}\right\|_{\max }\left(\frac{h_{\tau_{i}}}{h_{i, \min }}\right)^{5+s-r} h_{\tau_{i}}^{r-m}\right) \\
\leq & C_{3} h_{\tau_{i}}^{r-m}\left\|\left(f_{n, r}\right)_{n \in \iota_{i}}\right\|_{\max }
\end{aligned}
$$

where $C_{3}$ only depends on the local quasi uniformity, i.e., on $\kappa$. This leads to

$$
\begin{aligned}
\left\|u_{\Delta}\right\| & \leq\left\|u_{\text {aux }}\right\|+\left\|u_{\Delta}-u_{\text {aux }}\right\| \\
& \leq C_{3}\|\mathbf{f}\|_{\text {max }}+\left\|u_{\Delta}-u_{\text {aux }}\right\| .
\end{aligned}
$$

Proof of Case a. We employ [12, Theorem 2] and (A.7) to obtain

$$
\left\|u_{\Delta}-u_{\text {aux }}\right\| \leq C h^{4}\left\|u_{\text {aux }}^{(4)}\right\| \leq C\left(\frac{h}{h_{\min }}\right)^{4}\|\mathbf{f}\|_{\max } .
$$

To prove (A.2) we assume $f \in C^{\ell}(I)$ for some $0 \leq \ell \leq 4$. Then, well known properties of divided differences imply

$$
\max _{1 \leq i \leq N}\left\{h_{\tau_{i}}^{\ell-m}\left\|\left(f_{n, \ell}\right)_{n \in \iota_{i}}\right\|_{\max }\right\} \leq C h^{\ell-m}\left\|f^{(\ell)}\right\|
$$


and similarly as in (A.8) we get

$$
\left\|u_{\Delta}-u_{\text {aux }}\right\| \leq C h^{4}\left\|u_{\text {aux }}^{(4)}\right\| \leq C h^{\ell}\left\|f^{(\ell)}\right\|,
$$

where $C$ depends on the global shape regularity $h / h_{\min }$.

Proof of Case b. From [12, Corollary] we conclude that on an interval $\tau=\left[t_{i-1}, t_{i}\right]$ it holds

$$
\left\|u_{\Delta}-u_{\mathrm{aux}}\right\|_{C^{0}(\tau)} \leq \frac{1}{8} h_{\tau}^{2}\left(\frac{h_{\tau}^{2}}{8}\left\|u_{\mathrm{aux}}^{(4)}\right\|_{C^{0}(\tau)}+\max _{\ell \in\{i-1, i\}}\left\{\left|u_{\mathrm{aux}}^{(2)}\left(t_{\ell}\right)-u_{\Delta}^{(2)}\left(t_{\ell}\right)\right|\right\}\right) .
$$

The first term in the right-hand side of (A.10) can be estimated by using (A.7) with $m=4$

$$
\left(\frac{h_{\tau}^{2}}{8}\right)^{2}\left\|u_{\mathrm{aux}}^{(4)}\right\|_{C^{0}(\tau)} \leq C_{4}\left\|\left(f_{n}\right)_{n \in \iota_{i}}\right\|_{\max }
$$

where $C_{4}$, again, only depends on the local quasi uniformity, i.e., on $\kappa$. Let $\ell \in\{1, \ldots, N-1\}$ be such that

$$
h_{\ell}^{2}\left|d_{\ell}^{(2)}\right|=\left\|\mathbf{h}^{2} \mathbf{d}^{(2)}\right\|_{\max }
$$

with

$$
d_{i}^{(2)}:=u_{\mathrm{aux}}^{(2)}\left(t_{i}\right)-u_{\Delta}^{(2)}\left(t_{i}\right), \quad \mathbf{h}^{2} \mathbf{d}^{(2)}:=\left(h_{i}^{2} d_{i}^{(2)}\right)_{i=1}^{N-1}, \quad h_{i}:=\frac{h_{\tau_{i}}+h_{\tau_{i+1}}}{2} .
$$

Then, according to $[12,(2)]$, the differences $d_{i}^{(2)}$ satisfy the relation

$$
\alpha_{\ell} d_{\ell-1}^{(2)}+2 d_{\ell}^{(2)}+\left(1-\alpha_{\ell}\right) d_{\ell+1}^{(2)}=r_{\ell}
$$

with $\alpha_{\ell}=\frac{h_{\tau_{\ell}}}{h_{\tau_{\ell}}+h_{\tau_{\ell+1}}}$ and

$$
r_{\ell}:=\alpha_{\ell} u_{\mathrm{aux}}^{(2)}\left(t_{\ell-1}\right)+2 u_{\mathrm{aux}}^{(2)}\left(t_{\ell}\right)+\left(1-\alpha_{\ell}\right) u_{\mathrm{aux}}^{(2)}\left(t_{\ell+1}\right)-6\left[t_{\ell-1}, t_{\ell}, t_{\ell+1}\right] f .
$$

Multiplying (A.12) by $h_{\ell}^{2}$ leads to ${ }^{3}$

$$
\begin{aligned}
h_{\ell}^{2} r_{\ell} & =\alpha_{\ell}\left(\frac{h_{\ell}}{h_{\ell-1}}\right)^{2} h_{\ell-1}^{2} d_{\ell-1}^{(2)}+2 h_{\ell}^{2} d_{\ell}^{(2)}+\left(1-\alpha_{\ell}\right)\left(\frac{h_{\ell}}{h_{\ell+1}}\right)^{2} h_{\ell+1}^{2} d_{\ell+1}^{(2)} \\
& \geq h_{\ell}^{2} d_{\ell}^{(2)}\left(2-\kappa^{2}\right) .
\end{aligned}
$$

Hence,

$$
\left\|\mathbf{h}^{2} \mathbf{d}^{(2)}\right\|_{\max } \leq \frac{1}{2-\kappa^{2}}\left\|\mathbf{h}^{2} \mathbf{r}\right\|_{\max }
$$

From $[12,(2)]$ we conclude that

$$
\left|h_{i}^{2} r_{i}\right| \leq 2 h_{i}^{4}\left\|u_{\mathrm{aux}}^{(4)}\right\|_{C^{0}\left(\omega_{i}\right)} \stackrel{(\mathrm{A} .7)}{\leq} C_{5}\left\|\left(f_{n}\right)_{n \in \iota_{i}^{\star}}\right\|_{\max }
$$

\footnotetext{
${ }^{3}$ From (A.3) it follows
}

$$
\frac{h_{\ell}}{h_{\ell-1}}=\frac{h_{\tau_{\ell+1}}+h_{\tau_{\ell}}}{h_{\tau_{\ell-1}}+h_{\tau_{\ell}}} \leq \frac{1+\kappa}{1+\frac{1}{\kappa}}=\kappa .
$$


with

$$
\omega_{i}:=\bigcup\left\{\tau \in \Delta \mid t_{i} \in \tau\right\} \quad \text { and } \quad \iota_{i}^{\star}:=\bigcup_{k: t_{i} \in \tau_{k}} \iota_{k} .
$$

Thus, the second term in (A.10) can be estimated by

$$
\frac{1}{8} h_{\tau}^{2} \max _{\ell \in\{i-1, i\}}\left\{\left|u_{\mathrm{aux}}^{(2)}\left(t_{\ell}\right)-u_{\Delta}^{(2)}\left(t_{\ell}\right)\right|\right\} \leq C_{6}\|\mathbf{f}\|_{\max }
$$

and (A.4) follows.

To prove (A.5) we assume $f \in C^{\ell}(I)$ for some $0 \leq \ell \leq 4$ and estimate next the first term in the right-hand side in (A.11) by using (A.7)

$$
\left(\frac{h_{\tau}^{2}}{8}\right)^{2}\left\|u_{\mathrm{aux}}^{(4)}\right\|_{C^{0}(\tau)} \leq C_{7} h^{\ell}\left\|f^{(\ell)}\right\| .
$$

For the second term in (A.10) we first observe that

$$
\max _{1 \leq i \leq N-1}\left|h_{i}^{2} r_{i}\right| \leq 2 \max _{1 \leq i \leq N-1} h_{i}^{4}\left\|u_{\text {aux }}^{(4)}\right\|_{C^{0}\left(\omega_{i}\right)} \stackrel{(\text { A.7) }}{\leq} C h^{\ell}\left\|f^{(\ell)}\right\|
$$

holds so that the second term in (A.10) can be estimated by

$$
\frac{1}{8} h_{\tau}^{2} \max _{\ell \in\{i-1, i\}}\left\{\left|u_{\text {aux }}^{(2)}\left(t_{\ell}\right)-u_{\Delta}^{(2)}\left(t_{\ell}\right)\right|\right\} \leq C h^{\ell}\left\|f^{(\ell)}\right\|
$$

where $C$ only depends on the local quasi-uniformity of the mesh and (A.4) follows.

\section{References}

[1] R.P. Agarwal, D. O'Regan, P.J.Y. Wong, Positive Solutions of Differential, Difference and Integral Equations, Kluwer Academic, Dordrecht, 1999.

[2] J. Appell, P.P. Zabrejko, Nonlinear Superposition Operators, Cambridge Tracts in Math., vol. 95, Cambridge Univ. Press, 1990.

[3] J. Banas, K. Goebel, Measures of Noncompactness in Banach Spaces, in: Lecture Notes in Pure and Applied Mathematics, vol. 60, Marcel Dekker, New York and Basel, 1980.

[4] J. Banas, A. Martinon, On monotone solutions of a quadratic integral equation of Volterra type, Comput. Math. Appl. 47 (2004) 271-279.

[5] J. Banas, L. Olszowy, Measures of noncompactness related to monotonicity, Comment. Math. 41 (2001) 13-23.

[6] J. BAnas, B. RzePkA, On existence and asymptotic stability of solutions of a nonlinear integral equation, J. Math. Anal. Appl. 284 (2003) 165-173.

[7] J. Banas, K. Sadarangani, Monotonicity properties of the superposition operator and their applications, J. Math. Anal. Appl. 340 (2008) 1385-1394. 
[8] R. L. Burden, J. D. Faires, Numerical Analysis, 8th Edition, Cengage Learning, 2005

[9] T.A. Burton, Volterra Integral and Differential Equations, Academic Press, New York, 1983.

[10] G. F. CARey, H. T. Dinh, Grading functions and mesh redistribution, SIAM J. NUMER. ANAL. Vol. 22, No. 5 (1985) 1028-1040.

[11] K. Deimling, Nonlinear Functional Analysis, Springer-Verlag, Berlin, 1985.

[12] D. Kershaw. A note on the convergence of interpolatory cubic splines. SIAM J. Numer. Anal., 8:67-74, 1971.

[13] K. Maleknejad, K. Nouri, R. Mollapourasl, Existence of solutions for some nonlinear integral equations, Communications in Nonlinear Science and Numerical Simulation, 14 (2009) 2559-2564.

[14] K. Maleknejad, K. Nouri, R. Mollapourasl, Investigation on the existence of solutions for some nonlinear functional-integral equations, Nonlinear Analysis 71 (2009) 1575-1578.

[15] K. Maleknejad, R. Mollapourasl, K. Nouri, Study on existence of solutions for some nonlinear functionalï $\frac{1}{2}$ integral equations, Nonlinear Analysis 69 (2008) 2582-2588.

[16] K. Maleknejad, P. Torabi, R. Mollapourasl, Fixed point method for solving nonlinear quadratic Volterra integral equations, Computers and Mathematics with Applications 62 (2011) 2555-2566.

[17] J. H. Mathews, K. D. Fink, Numerical Methods Using Mathlab, 4th edition, Prentice-Hall Inc. New Jersey, USA, 2004.

[18] D. O'Regan, M. Meehan, Existence theory for nonlinear integral and integro-differential equations, Kluwer Academic, Dordrecht, 1998.

[19] J. Stoer, R. Bulirsch, Introduction to numerical analysis, Third edition, Springer-Verlag, New York, 2002.

[20] P. G. Todorov. New explicit formulas for the $n$th derivative of composite functions. Pacific J. Math., 92(1):217-236, 1981. 